\title{
Correlating Size and Composition-Dependent Effects with Magnetic, Mössbauer, and PDF Measurements in a Family of Catalytically Active Ferrite Nanoparticles
}

Amanda L. Tiano ${ }^{1}$, Georgia C. Papaefthymiou ${ }^{2,3}$, Crystal S. Lewis ${ }^{1}$, Jinkyu Han $^{4}$ Cheng Zhang ${ }^{4}$, Qiang $\mathrm{Li}^{4}$, Chenyang Shi, ${ }^{5}$ A. M. Milinda Abeykoon, ${ }^{6}$ Simon J. L. Billinge, ${ }^{4,5}$ Eric Stach, ${ }^{7}$ Justin Thomas ${ }^{1}$, Kevin Guerrero ${ }^{1}$, Pablo Munayco ${ }^{3}$, Jimmy Munayco ${ }^{3}$, Rosa B. Scorzelli ${ }^{3}$, Philip Burnham ${ }^{2}$, Arthur J. Viescas ${ }^{2}$, and Stanislaus S. Wong ${ }^{1,4^{*}}$

${ }^{1}$ Department of Chemistry, State University of New York at Stony Brook, Stony Brook, NY 11794-3400

${ }^{2}$ Department of Physics, Mendel Hall, Villanova University, 800 Lancaster Avenue, Villanova, PA 19085

${ }^{3}$ Centro Brasileiro de Pesquisas Físicas, Rua Xavier Sigaud 150, 222909-180

Rio de Janeiro, Brazil

${ }^{4}$ Condensed Matter Physics and Materials Sciences Department, Building 480, Brookhaven National Laboratory, Upton, NY 11973

${ }^{5}$ Department of Applied Physics and Applied Mathematics, Columbia University, New York, NY 10027

${ }^{6}$ Photon Sciences Directorate, Building 741, Brookhaven National Laboratory, Upton, NY 11973

${ }^{7}$ Center for Functional Nanomaterials, Building 735, Brookhaven National Laboratory, Upton, NY 11973

*To whom correspondence should be addressed. Email: Stanislaus.wong@stonybrook.edu; sswong@bnl.gov. Phone: 631-632-1703; 631-344-3178.

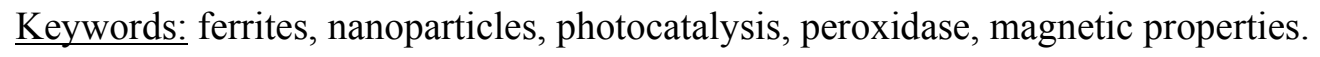


Abstract: $T$ The magnetic spinel ferrites, $\mathrm{MFe}_{2} \mathrm{O}_{4}$ (wherein ' $\mathrm{M}$ ' $=$ a divalent metal ion such as but not limited to $\mathrm{Mn}, \mathrm{Co}, \mathrm{Zn}$, and $\mathrm{Ni}$ ), represent a unique class of magnetic materials in which the rational introduction of different 'M's can yield correspondingly unique and interesting magnetic behaviors. Herein we present a generalized hydrothermal method for the synthesis of single-crystalline ferrite nanoparticles with ' $\mathrm{M}$ ' $=\mathrm{Mg}, \mathrm{Fe}, \mathrm{Co}, \mathrm{Ni}, \mathrm{Cu}$, and $\mathrm{Zn}$ ), which can be systematically and efficaciously produced simply by changing the metal precursor. Our protocol can moreover lead to reproducible size control by judicious selection of various surfactants. As such, we have probed the effects of both (i) size and (ii) chemical composition upon the magnetic properties of these nanomaterials using complementary magnetometry and Mössbauer spectroscopy techniques. The structure of the samples was confirmed by atomic PDF analysis of X-ray and electron powder diffraction data as a function of particle size. These materials retain the bulk spinel structure to the smallest size (i.e. $3 \mathrm{~nm}$ ). In addition, we have explored the catalytic potential of our ferrites as both (a) magnetically recoverable photocatalysts and (b) biological catalysts, and noted that many of our asprepared ferrite systems evinced intrinsically higher activities as compared with their iron oxide counterparts. 


\section{Introduction}

Magnetic nanomaterials have become an area of considerable focus, due to their unique physicochemical properties by contrast with their bulk counterparts. Specifically, the so-called metal ferrites, $\mathrm{MFe}_{2} \mathrm{O}_{4}$ (wherein ' $\mathrm{M}$ ' $=\mathrm{Co}, \mathrm{Zn}, \mathrm{Ni}$, and $\mathrm{Mn}$ ), for example, represent a well known and unique class of materials, which have long been studied for their novel magnetic and electronic properties. $\mathrm{MFe}_{2} \mathrm{O}_{4}$ possesses a cubic spinel structure in which the oxygen atoms are placed in a cubic close-packed arrangement with the metal atoms residing at tetrahedral and octahedral sites, labeled as (A) and [B] sites, respectively. ${ }^{1,2}$ It is known that altering the occupancies of the (A) and [B] sites can give rise to slight variations of the spinel structure, which can affect the corresponding nature and magnitude of superexchange interactions, ${ }^{3}$ namely $\mathrm{A}-\mathrm{A}, \mathrm{B}-\mathrm{B}$, and $\mathrm{A}-\mathrm{B}$, of which the $\mathrm{A}-\mathrm{B}$ interactions tend to be highly significant. Exhibiting reliable control over the distribution of $\mathrm{M}^{2+}$ and $\mathrm{Fe}^{3+}$ cations in each site should result in an intrinsically magnetically tunable system. ${ }^{1}$ Hence, by choosing either the appropriate $\mathrm{M}^{2+}$ ion or mixture of $\mathrm{M}^{2+}$ ions, a wide variety of magnetic behaviors can be observed.

Specifically, below $20 \mathrm{~nm}$ in size, ferrite nanoparticles display size-dependent superparamagnetic behavior as well as high saturation magnetization. ${ }^{4}$ In addition, the metal ferrites exhibit high electrical resistivity, high permeability, and excellent chemical stability, thereby rendering them as ideal candidates for electromagnetic applications, spanning both high frequency and wide temperature ranges. ${ }^{1}$ Therefore, by combining these properties with the advantages of nanoscale materials (e.g. small sizes, high surface area-to-volume ratios, and often tunable size-dependent properties), ferrite nanostructures inherently possess multiple advantages over other magnetic materials and are also generally lower in cost, thereby enhancing their potential in numerous, wide-ranging applications. Specifically, ferrites have been investigated as active materials for biomedicinal devices, ${ }^{5-7}$ sensors, ${ }^{8,9}$ batteries, ${ }^{10}$ magnetic energy storage, ${ }^{11}$ components for electronics, ${ }^{9,}{ }^{12}$ magnetically 
recoverable catalyst supports, ${ }^{13-16}$ photocatalysts, ${ }^{17-19}$ and magnetic resonance imaging (MRI) contrast agents. 5,20

The key to tailoring ferrite nanomaterials for actual real-world applications is by either creating new or modifying existing synthetic protocols to precisely control the size, composition, crystallinity, and morphology of these nanomaterials in order to generate dependable structure-property correlations. Such a strategy entails developing a synthetic methodology, which is not only facile but also reproducible with a number of independent reaction variables which can be reliably adjusted, so as to yield a desired, tailored product.

To date, there have been numerous methods utilized for the synthesis of nanoscale motifs of $\mathrm{MFe}_{2} \mathrm{O}_{4}$. These have included methodologies based on hydrothermal ${ }^{10,21}$ and solvothermal techniques, ${ }^{22-24}$ thermal decomposition, ${ }^{2,5,7,20,25-28}$ co-precipitation, ${ }^{4,29-31}$ electrochemical manipulation, ${ }^{32}$ microemulsion, ${ }^{1,33,34}$ and other solution-based treatments (i.e. sol-gel, polyol, and so forth). ${ }^{35-41}$ However, an overwhelming majority of the existing literature is primarily associated with developing workable protocols for the production of a single spinel ferrite. As such, there are relatively few generalized and established methods that have been practically applied to the controlled synthesis of broad classes of ferrites of various chemical compositions. . $^{2,5,20,22-24,28}$

Herein our contribution has been to devise a generalized hydrothermal method (Section I) for the reproducible synthesis of pure single-crystalline $\mathrm{MFe}_{2} \mathrm{O}_{4}$ nanoparticles, wherein ' $\mathrm{M}$ ' $=\mathrm{Mg}, \mathrm{Co}, \mathrm{Ni}, \mathrm{Cu}, \mathrm{Zn}$, and even $\mathrm{Fe}$, in which a simple variation of the metal precursor permits for the corresponding production of ferrites of various chemical compositions. We have moreover investigated the role of reaction time, choice of precursor, temperature, $\mathrm{pH}$, and surfactants and correlated their individual effects with the corresponding degree of control over the size, crystallinity, and morphology of our as-prepared nanoparticles. Of the reaction parameters analyzed, the choices of precursor and inclusion of surfactants were determined to have most markedly affected the particle composition, phase 
purity, and size, respectively. In addition, unlike prior reports, we also noted that a relatively long reaction time ( $\geq 12$ hours) was not critical and for instance, we were able to significantly reduce the overall reaction time to 3 hours in the case of zinc ferrites.

The key contribution of our work is that we include a comprehensive set (Section II) of magnetic characterization data from Superconducting Quantum Interference Device (SQUID) vibrating sample magnetometry and Mössbauer spectroscopy collected on our family of ferrite nanoparticles as a function of morphology, physical size, and chemical composition, to an extent rarely attempted prior to this current study. Finally, in Section III, we carry out a detailed structural study of the nanomaterials in an attempt to relate the magnetic properties to structural features. The structural analysis combines (i) an atomic pair distribution function (PDF) analysis of X-ray powder diffraction data collected at the National Synchrotron Light Source at Brookhaven National Laboratory (BNL) with (ii) an electron PDF (ePDF) analysis ${ }^{42}$ using electron diffraction data obtained at the Center for Functional Nanomaterials at BNL.

In addition to structural characterization, we have investigated the potential of our ferrite nanoparticles as catalysts (Section IV) for peroxidase-like applications and as magnetically separable photocatalysts. In the presence of methylene blue, we investigated the photocatalytic potential of our ferrite nanoparticles under UV irradiation. These experiments revealed that the photocatalysis process is composition-dependent, with $\mathrm{Mg}$ ferrite nanoparticles as the most active of the series studied herein. Moreover, we find that our nanoparticles display enhanced activities even at concentrations of $0.25 \mathrm{~g} / \mathrm{L}$, a level which is significantly less than the $1.0 \mathrm{~g} / \mathrm{L}$ concentrations previously reported in the literature.

Furthermore, we also determined that many ferrite nanoparticles exhibit peroxidaselike activity towards a typical, conventional substrate. Specifically, $\mathrm{Mg}, \mathrm{Co}$, and $\mathrm{Cu}$ ferrite nanoparticles yielded higher catalytic activities by comparison with iron oxide. Hence, we have highlighted the multifaceted potential of our as-prepared nanostructures, because, in 
addition to their robust magnetic properties, our ferrite nanoparticles are also excellent catalysts, with specific chemical compositions consistently yielding intrinsically higher activities. In essence, our novelty herein has been to demonstrate an unprecedented understanding of structure-property correlations in as-prepared nanoscale ferrites.

\section{Experimental}

Materials and Reagents. For the metal precursors themselves, different variations were tried, depending on the desired metal ferrite and size. Specifically, we used cobalt (II) chloride (anhydrous, $97 \%$, Aldrich), cobalt chloride heptahydrate (98\%, Aldrich), zinc (II) fluoride (99\%, Aldrich), zinc chloride (Aldrich), copper chloride (Baker), copper (II) nitrate trihydrate (98\%, Alfa Aesar), magnesium boride (Alfa Aesar), magnesium sulfate (MallinckrodtBaker), magnesium nitrate (analytical reagent, Mallinckrodt), nickel (II) sulfate hexahydrate (Acros Organics), nickel acetylacetonate (95\%, Sigma Aldrich), nickel chloride hexahydrate (Baker), nickel fluoride (99\%, Aldrich), nickel oxalate (99.9985\% metals basis, Alfa Aesar), magnesium chloride (98\%, Aldrich), iron (II) chloride tetrahydrate (99\%, Aldrich), and anhydrous iron (III) chloride (98 \%, EM Science) to synthesize cobalt, zinc, copper, magnesium, nickel, and iron ferrites, respectively.

In addition, as solvents, surfactants, as well as catalytic substrates and reagents for our synthesis and catalysis experiments, we also utilized ethanol $(99.5+\%, 200$ proof, anhydrous, Acros Organics), toluene (ACS reagent, Acros Organics), dimethyl sulfoxide $(99.8+\%$, anhydrous, Alfa Aesar), meso-2,3-dimercaptosuccinic acid (97\%, Alfa Aesar), 3aminopropyltriethoxysilane (APTES, 99 \%, Acros Organics), Igepal CO-630 (Aldrich), polyvinylpyrrolidone with $\mathrm{M}_{\mathrm{w}}=1300000$ (Acros Organics), Tergital NP-9 surfactant (polyoxyethylene(9)nonylphenyl ether, Aldrich), dextran (from Leuconostoc spp., $\mathrm{M}_{\mathrm{w}}=5$ 000, Sigma Life Sciences), Triton X-100 (EMD Industries), sodium hydroxide (99 \%, EMD Science), methylene blue (Mallinckrodt-Baker), hydrogen peroxide (30\% by weight aqueous 
solution, Sigma Aldrich), glacial acetic acid (Mallinckrodt-Baker), sodium acetate trihydrate (Mallinckrodt-Baker), and 3,3',5,5'-tetramethylbenzidine (liquid TMB substrate, SigmaAldrich). All of these reagent chemicals were used, as received, without any additional purification steps involved.

Methodology. $\mathrm{MFe}_{2} \mathrm{O}_{4}$ (where ' $\mathrm{M}$ ' $=\mathrm{Mg}, \mathrm{Fe}, \mathrm{Co}, \mathrm{Ni}, \mathrm{Cu}$, and $\mathrm{Zn}$ ) nanoparticles were prepared by using a hydrothermal method, appropriately modified from previous work by Wang et al. which had focused on the synthesis of $\mathrm{MnFe}_{2} \mathrm{O}_{4}$ nanorods. ${ }^{21}$ Specifically, a $0.5 \mathrm{M}$ solution of the desired metal precursor (e.g. $\mathrm{CoCl}_{2}, \mathrm{NiSO}_{4}, \mathrm{ZnF}_{2}, \mathrm{Cu}\left(\mathrm{NO}_{3}\right)_{2} \cdot 3 \mathrm{H}_{2} \mathrm{O}, \mathrm{MgB}_{2}, \mathrm{FeCl}_{2} \cdot 4 \mathrm{H}_{2} \mathrm{O}$, or $\mathrm{FeCl}_{3}$, depending on the desired ferrite composition) in $16 \mathrm{~mL}$ of water was mixed thoroughly by magnetic stirring in a beaker using a micro (flea) type stir bar at $~ 300 \mathrm{rpm}$. Subsequently, $\mathrm{FeCl}_{3}$ was added to the solution in order to create a $0.1 \mathrm{M}$ solution. It is important to note that the molarities of the precursor solutions were calculated by taking into account of the volume of the final solution, namely $18.3 \mathrm{~mL}$, in order to fill the autoclave to $80 \%$ of its capacity. Finally, $2.3 \mathrm{~mL}$ of a $4.4 \mathrm{M}$ solution of $\mathrm{NaOH}$ was added to the beaker under vigorous magnetic stirring.

After these steps, the progression of the reaction was accompanied by a visible color change from orange/yellow to a dark brown/black color, which varied, depending on the metal precursor employed. The solution was allowed to stir using a micro (flea) type stir bar at $\sim 300 \mathrm{rpm}$ for 30 minutes after which it was transferred to a $23 \mathrm{~mL}$ Teflon-lined stainless steel autoclave (Parr Instruments) and subsequently sealed. In a typical synthesis, the autoclave was placed in an oven and heated to $190^{\circ} \mathrm{C}$ for 3 - 12 hours. In order to control the ferrites' size regime, surfactants such as NP9 and APTES were employed (Tables 1 and S1) at the reaction temperatures, mentioned previously. For crystalline ultra-small ferrites in particular, the autoclave was heated to $220^{\circ} \mathrm{C}$ for 12 hours, and then allowed to cool naturally to room temperature. The product was subsequently washed four times with aliquots (i.e. 1 aliquot is 
$\sim 10 \mathrm{~mL}$ ) of distilled water and once with an aliquot of ethanol, isolated by centrifugation, decantation, and finally placed in a drying oven at $\sim 80^{\circ} \mathrm{C}$ to remove residual solvent, thereby yielding $\sim 150 \mathrm{mg}$ of sample. As previously mentioned, variations in reaction time, temperature, and $\mathrm{pH}$ were explored, and the details of these findings are presented in the Results and Discussion section and the Supporting Information section (Figures S1 - S4) as well as summarized in Tables 1 and $\mathrm{S} 1$.

Structural Characterization. Powder samples for X-ray diffraction (XRD) were prepared using sonicated slurries of the product in absolute ethanol, deposited onto glass slides, and allowed to dry under ambient conditions. XRD analysis was conducted using a Scintag diffractometer with $\mathrm{Cu} \mathrm{K} \alpha$ radiation $(\lambda=1.54 \AA)$, scanned with a $1^{\circ}$ step size over a range of $20^{\circ} \leq 2 \theta \leq 80^{\circ}$ in the Bragg configuration.

For transmission electron microscopy (TEM) analysis, as-prepared samples were prepared by first dispersing in ethanol, followed by placing a drop of that dilute solution onto a lacey carbon or ultrathin carbon grid (Ted Pella). We should note that the $\mathrm{MgFe}_{2} \mathrm{O}_{4}$ nanoparticles (sample $\mathrm{Mg} 1$ ) in particular were ultrasonicated for 90 min and ground with a mortar and pestle for 30 additional min in order to achieve a relatively more homogeneous sample. The samples were then dried at room temperature under air, until all of the ethanol had evaporated. The initial morphology and sizes of the final products were investigated using a FEI-Tecnai12 BioTwinG ${ }^{2}$ instrument for low-resolution imaging. Size measurements were collected from a minimum of 50 particles for each sample in order to compute their average and standard deviation. High-resolution transmission electron microscopy (HRTEM) and selected-area electron diffraction (SAED) analyses were performed using a JEOL 3000F instrument at accelerating voltages of $300 \mathrm{kV}$.

For energy dispersive X-ray spectroscopy (EDS), samples were dispersed in ethanol, sonicated to ensure reasonable uniformity and homogeneity, and then deposited onto a pre- 
cleaned silicon wafer. The samples were secured to an aluminum bar with either conductive carbon or copper tape. The elemental analysis was conducted using a field-emission scanning electron microscope (FE-SEM Leo 1550), operating at an accelerating voltage of $20 \mathrm{kV}$, and equipped with EDS capabilities.

Samples prepared for BET analysis were initially dried in an oven overnight to remove any residual solvents. Subsequently, $\sim 100 \mathrm{mg}$ of material was placed in an analysis tube and degassed for 2 hours at $150^{\circ} \mathrm{C}$ in order to remove any adsorbed gases on the particle surfaces. After degassing, the BET surface area analysis was conducted with a Quantachrome Nova 2200e Series Instrument with a 10-point sampling method.

Spectroscopy. Optical absorption in the far-infrared (far-IR) region was measured for the asprepared ferrite samples using a Nexus 670 FT-IR (Thermo Nicolet) instrument, equipped with a single-reflectance zinc selenide ( $\mathrm{ZnSe}$ ) attenuated total reflectance (ATR) accessory, a solid substrate beam splitter, and a DTGS polyethylene detector. These as-prepared solid powders were placed onto the $\mathrm{ZnSe}$ crystal and measurements were obtained in transmittance mode using the Smart Performer module. The data shown represent an average of 64 accumulated spectra. Absorption in the UV-Visible region was measured on the solutions of each ferrite nanoparticle dispersed in ethanol. The measurements were taken with a UV1 UVVisible spectrophotometer (Thermo Scientific).

Magnetic and Mössbauer Characterization. Magnetic data were taken on powders of ferrite nanoparticles, which were encapsulated in paraffin wax within a gelatin capsule placed into a plastic straw in order to allow for immersion into the magnetometer. Magnetization measurements were then taken with a Quantum Design Magnetic Property Measurement System (MPMS) at fields of up to $5 \mathrm{~T}$ and temperatures between 2 and $300 \mathrm{~K}$. For Mössbauer spectroscopy, at room temperature (RT), samples were studied, as received, by dispersing in boron nitride powder. Transmission geometry experiments were performed at a constant acceleration mode. Two different spectrometers were used, namely a 1024 channel SEE Co., 
and a 512 channel Halder spectrometer with ${ }^{57} \mathrm{Co}$ radioactive sources embedded in rhodium. Both spectrometers were equipped with Janis Research Co. cryogenic dewars for sample temperature variation from $4.2 \leq \mathrm{T} \leq 300 \mathrm{~K}$. The spectrometers were calibrated with a $6-\mu \mathrm{m}$ thick metal iron foil enriched in ${ }^{57} \mathrm{Fe}$ as the standard absorber. All measurements were performed at high velocity $( \pm 12 \mathrm{~mm} / \mathrm{s})$. Data were collected as counts against source velocity in $\mathrm{mm} / \mathrm{s}$. Spectral fits were carried out using either the WMOSS software package licensed by SEE Co. or the NORMOS code (Brand 1995) software package licensed by Wissel Instruments Inc. Spectral analysis was based on a least-square fitting algorithm, assuming each spectrum to be a sum of Lorentzian absorption lines grouped into quadrupole doublets and magnetic sextets, including a distribution of hyperfine fields and intermediate relaxation spectra. The relative amounts of each constituent present were determined by measuring the areas under the relevant spectral peaks, assuming equal recoil-free fractions. The isomer shifts have been given, relative to $\alpha-\mathrm{Fe}$ at RT.

Atomic Pair Distribution Function (PDF) Analysis. PDF data were obtained from synchrotron X-ray data taken on various ferrite nanoparticles (i.e. Co and Ni ferrites), encapsulated in kapton capillary tubes with diameter $1.0 \mathrm{~mm}$, sealed at both ends, and measured at $100 \mathrm{~K}$ under nitrogen flow. Synchrotron X-ray total scattering experiments were conducted at the beamline X17A at the National Synchrotron Light Source (NSLS) at Brookhaven National Laboratory. The rapid acquisition pair distribution function (RaPDF) technique ${ }^{43}$ was applied using a large area 2-D Perkin Elmer detector $(2084 \times 2084$ pixels and $200 \times 200 \mu \mathrm{m}$ pixel size), mounted orthogonal to the beam path with a sample-to-detector distance of $208.131 \mathrm{~mm}$ for Co and Ni ferrite samples, with an incident X-ray energy of $67.42 \mathrm{keV}(\lambda=0.1839 \AA)$. The raw $2 \mathrm{D}$ data were azimuthally integrated and converted to $1 \mathrm{D}$ intensity versus $2 \theta$ using FIT2D program, ${ }^{44}$ where $2 \theta$ is the angle between the incident and the scattered X-ray beams. The PDFget $\mathrm{X} 3^{45}$ program was used to correct and normalize the raw diffraction data and then Fourier transform them to obtain the PDF, $G(r)$, according to Equation E1, 


$$
G(r)=\frac{2}{\pi} \int_{Q_{\min }}^{Q_{\max }} Q[S(Q)-1] \sin Q r d Q
$$

Here, $G(r)$ gives the probability of finding a pair of atoms separated by a distance of $r$, $Q$ is the magnitude of the momentum transfer on scattering and $Q=4 \pi \sin \theta / \lambda$, and $S(Q)$ is the properly corrected and normalized powder diffraction intensity measured from $Q_{\min }$ to $Q_{\max }{ }^{46}$ Moreover, to calibrate the sample-to-detector distance and to determine the $Q_{\text {damp }}$ and $Q_{\text {broad }}$, the parameters that correct the PDF envelope function for instrument resolution effects, nickel was also measured as the standard material. ${ }^{47,48}$ The refined values for $Q_{\text {damp }}$ and $Q_{\text {broad }}$ were $0.0407 \AA^{-1}$ and $0.0185 \AA^{-1}$, respectively. They were fixed in the subsequent model fits to the PDF data of each nanoparticle sample.

We also used the recently demonstrated ePDF method ${ }^{42}$ on a subset of the smallest nanoparticles of $\mathrm{Zn}$ and $\mathrm{Ni}$ ferrite. The nanoparticles were dispersed on electron microscope grids. Then the diffraction data were collected using a $20 \mathrm{keV}$ electron beam on a JEOL 1400, utilizing a Gatan CCD camera with a $14 \mu \mathrm{m}$ pixel and a 2048 x 2048 viewing area. A diffraction pattern of a Si calibration standard sample of known lattice parameter was measured prior to the samples of interest (SOI). Then the calibration sample was replaced with the SOI, which were measured without changing any of the experimental parameters. The camera length calibration was performed using the software package, Fit $2 \mathrm{D},{ }^{44}$ which has built-in features for performing the calibration. The Debye-Scherrer rings from the Si calibration standard were fit with the known $d$-spacing fixed but allowing for the camera length and detector non-orthogonality to vary. The 2D diffraction images from SOI were reduced to 1D diffraction patterns using Fit2D, and then further transformed to ePDFs using an in-house program, i.e. PDFgetE. ${ }^{49}$

The ferrite samples share the general formula $\left(\mathrm{A}_{1-\mathbf{x}} \mathrm{Fe}_{\mathbf{x}}\right)\left[\mathrm{A}_{\mathbf{x}} \mathrm{Fe}_{2-\mathbf{x}}\right] \mathrm{O}_{4}$, where round and square brackets denote the tetrahedral and octahedral sites of the spinel structure, respectively. Here, ' $x$ ' represents the degree of inversion (DOI). The PDF modeling was carried out using the SrFit program. ${ }^{50}$ The structural model of a cubic spinel with the space group of $F d \overline{3} m$ 
(No. 227) was used. The fractional coordinates for tetrahedral and octahedral sites are at (1/8, $1 / 8,1 / 8)$ and $(1 / 2,1 / 2,1 / 2)$, respectively, while $\mathrm{O}$ atoms are located on $(z, z, z)$. For the Co and Ni samples, the DOIs were derived from previous Mössbauer experiments and were applied in the model fits. The ePDFs from the small nanoparticles were also modeled using the same approach in the SrFit program and the same structural models.

In order to estimate the crystallite size, or the range of structural coherence of the samples, we first assume they are of spherical shape and then we consider PDFs of nanoparticles as the attenuated bulk PDF $\mathrm{G}_{\mathrm{Bu}} \mathrm{llk}_{\mathrm{k}}(\mathrm{r})$ by an envelope function $\gamma_{0}(\mathrm{r})$ as follows, according to Equation E2: $51-53$

$$
\mathrm{G}(\mathrm{r})=\mathrm{G}_{\mathrm{Bulk}}(\mathrm{r}) \gamma_{\mathrm{o}}(\mathrm{r})
$$

where $\gamma_{0}(r)$ is expressed as

$$
\gamma_{0}(\mathrm{r})=\left[1-\frac{3 \mathrm{r}}{2 \mathrm{D}}+\frac{1}{2}\left(\frac{\mathrm{r}}{\mathrm{D}}\right)^{3}\right] \theta(\mathrm{D}-\mathrm{r})
$$

and a Heaviside step function ensures $\gamma_{0}(\mathrm{r})$ is zero beyond the diameter of the nanoparticle,

$$
\theta(D-r)=\left\{\begin{array}{lll}
0 & D & <r \\
1 & D & >
\end{array}\right.
$$

Photocatalysis. First, a Beer's law plot of methylene blue was determined using aqueous solutions, ranging from $0.05-5 \mathrm{mg} / \mathrm{L}$ in concentration. For the photodegradation experiments, $25 \mathrm{mg}$ of the desired ferrite nanoparticles have been added to a beaker containing $100 \mathrm{~mL}$ of a $5 \mathrm{mg} / \mathrm{L}$ aqueous solution of methylene blue, such that the ferrites maintain a concentration of $0.25 \mathrm{~g} / \mathrm{L}$. The solution was stirred magnetically at 400 r.p.m. in order to establish an absorption/desorption equilibrium between the dye and the nanoparticles. The solution was then irradiated at $366 \mathrm{~nm}$ with an Entela UVGL-58 Mineralight UV lamp $(115 \mathrm{~V} \sim 60 \mathrm{~Hz}$, 
0.16 Amps), placed at a distance of $\sim 2 \mathrm{~cm}$ from the beaker. Aliquots were taken at hourly intervals, and then subjected to centrifugation at 7000 r.p.m. in order to separate the nanoparticles from the dye. The supernatant was transferred to a quartz cell, and the optical absorbance was measured over the range of 200 - $800 \mathrm{~nm}$ with a UV1 UV-Visible spectrophotometer (Thermo Scientific). The absorbance at $664 \mathrm{~nm}$ was utilized to calculate the dye concentrations from a linear fit of the Beer's law plot. The final concentration over the initial concentration $\left(\mathrm{C} / \mathrm{C}_{0}\right)$ was plotted with respect to time to elucidate the reaction order.

Peroxidase-like activity. All experiments were carried out at room temperature in a $3.5 \mathrm{~mL}$ quartz cuvette. Aqueous solutions of the ferrite nanoparticles were prepared at $1 \mathrm{mg} / \mathrm{mL}$ concentrations in a sodium acetate buffer $(0.2 \mathrm{M}, \mathrm{pH}$ 4) solution. $1 \mathrm{~mL}$ of ferrite solution was mixed with $1 \mathrm{~mL}$ of $30 \% \mathrm{H}_{2} \mathrm{O}_{2}$ and $1 \mathrm{~mL}$ of the liquid TMB substrate. Upon addition of the generally colorless TMB, a blue color change was observed. Subsequent changes in absorbance, denoting reaction progression, were monitored in rate mode at $652 \mathrm{~nm}$ (the maximum absorption wavelength of $\mathrm{TMB}^{+\bullet}$, a blue one-electron oxidation product representing the cationic free radical analogue of TMB) as a function of time, using a UV1 UV-Visible spectrophotometer (Thermo Scientific).

\section{Results and Discussion}

We systematically investigated several reaction parameters in our hydrothermal synthesis of the spinel ferrites, including reaction time, reaction temperature, the nature of the precursor, concentration of $\mathrm{NaOH}$, and the nature of the surfactants. In our synthesis approach, we found that we could exhibit control over ferrite composition mainly by changing the identity of the metal precursor. Additionally, by utilizing surfactants, specifically Igepal CO-630 (NP-9) and 3-aminopropyltriethoxysilane (APTES), we were able to reliably control particle size. When employing APTES in particular, we were able to generate reasonably 
monodisperse ultra-small nanoparticles (i.e. measuring $\leq 4.0 \mathrm{~nm}$ in diameter) for a series of $\mathrm{Co}, \mathrm{Ni}$, and Zn metal ferrites.

The respective roles of various synthesis parameters such as reaction temperature, reaction time, base concentration, and surfactant were systematically analyzed, highlighted, and ultimately discussed in the Supplementary section (Figures S1, S2, S3, and S4). Specifics for each individual reaction we tried can be found in Table S1. The details for the most relevant and salient products used for our subsequent characterization and catalysis experiments are highlighted in Table 1. Of the experimental parameters probed, we found that (a) the nature of the precursor as well as (b) the surfactant yielded the most significant effect upon the resulting product chemical composition and size, respectively. The effects of all individual parameters tested and the corresponding outcomes on the resulting products yielded are discussed in exhaustive detail in the Supporting Information section.

\section{Product Characterization - Size, composition, and morphology of as-generated ferrites}

In our efforts to generate our 'family' of ferrite systems, we systematically tested out various precursors of different transition metals (Table S1) under standard synthetic conditions (i.e. $190^{\circ} \mathrm{C}$ for $12 \mathrm{~h}$ and in the absence of surfactant). In so doing, we were able to generate ferrite nanoparticles, possessing different chemical compositions. Specifically, we successfully synthesized reasonably pure, 'optimized' nanoparticles of $\mathrm{MgFe}_{2} \mathrm{O}_{4}, \mathrm{CoFe}_{2} \mathrm{O}_{4}$, $\mathrm{NiFe}_{2} \mathrm{O}_{4}, \mathrm{CuFe}_{2} \mathrm{O}_{4}$, and $\mathrm{ZnFe}_{2} \mathrm{O}_{4}$ with precursors of $\mathrm{MgB}_{2}, \mathrm{CoCl}_{2}, \mathrm{NiSO}_{4}$ (or $\mathrm{Ni}(\mathrm{acac})_{2}$ ), $\mathrm{Cu}\left(\mathrm{NO}_{3}\right)_{2} \cdot 3 \mathrm{H}_{2} \mathrm{O}$, and $\mathrm{ZnF}_{2}$, respectively, although other precursors were found to work as well in these syntheses to varying degrees. The corresponding, representative XRD data, shown in Figure 1, demonstrate that the samples can be readily indexed to the pure cubic spinel ferrites (space group: $\boldsymbol{F} d \overline{3} m$ ) with no readily detectable impurities, though we found $\mathrm{MgFe}_{2} \mathrm{O}_{4}$ to possess a minor impurity phase consisting of orthorhombic $\mathrm{Fe}_{2} \mathrm{O}_{3}$ (JCPDS \#726233). We also note that to synthesize pure iron oxide (i.e. magnetite, $\mathrm{Fe}_{3} \mathrm{O}_{4}$ ), $\mathrm{FeCl}_{2} \cdot 4 \mathrm{H}_{2} \mathrm{O}$ 
was specifically used as the precursor (samples Fe1 and Fe2, i.e. Figures S1B and S1E). By contrast, if $\mathrm{FeCl}_{3}$ were utilized as the precursor, as with the other ferrite systems synthesized, only large spheres of $\mathrm{Fe}_{2} \mathrm{O}_{3}$ (sample $\mathrm{Fe} 3$ ) could be generated (Figure $\mathrm{S} 1 \mathrm{~A}$ ).

Representative images of transmission electron microscopy (TEM) analysis of typical nanoparticles of metal ferrites, shown in Figure 2, revealed that the nanoparticles were reasonably uniform in size and shape. Subsequent investigation with high-resolution TEM (HRTEM) evinced that the as-prepared nanoparticles were single-crystalline in nature (Figure 3) with no visible defects or dislocations. The corresponding selected-area electron diffraction (SAED) patterns showed a ring-like pattern with relatively sharp discrete spots (Figure 3, respective insets), which are typical for single-crystalline materials, possessing a small particle size with respect to the diameter of the selected-area aperture. The visible rings in the SAED pattern could be indexed to the (111), (220), (311/222), (400), (422), (440), and (511) $h k l$ spacings, respectively, for cubic $\mathrm{MFe}_{2} \mathrm{O}_{4}$ with ' $\mathrm{M}$ ' representing a given metal.

In general, we noted that the presence of APTES and NP-9, a cationic and a non-ionic surfactant, respectively, could reduce the size of the ferrite nanoparticles. It is well known that the presence of surfactants can potentially alter the particle size of nanostructures by creating a barrier for mass transfer and correspondingly modulating the surface energy of coated nanoparticles, therefore potentially decreasing their surface tension. This can prevent nanoparticle agglomeration. Furthermore, for our specific reaction protocol, the dissolution of the metal precursors under basic conditions in $\mathrm{NaOH}$ allows for the formation of metal hydroxides, which can then intercalate between the $\mathrm{NH}_{3}{ }^{+}$groups of APTES so as to form ion pairs by electrostatic interactions. ${ }^{54}$ That is, the differential selective adsorption and interactions of both APTES and NP-9 with inorganic precursors can impact the nucleation process, and therefore, subsequently affect the resulting crystallite size.

Not surprisingly, TEM and HRTEM images of our crystalline ultra-small $\mathrm{Zn}$ and $\mathrm{Ni}$ ferrites prepared in the presence of APTES at higher reaction temperatures $\left(220^{\circ} \mathrm{C}\right)$ than 
conventional standard reaction temperatures $\left(190^{\circ} \mathrm{C}\right)$ are shown in Figure 4A-B and Figure $4 \mathrm{C}-\mathrm{D}$, respectively. On the basis of statistical measurements of several tens of nanoparticles, pertaining to each sample analyzed, the average diameters of our crystalline as-prepared $\mathrm{Zn}$ and Ni ferrites, i.e. the so-called "ultra-small nanoparticles", were noted to be $3.1 \pm 0.7 \mathrm{~nm}$ and $3.0 \pm 0.6 \mathrm{~nm}$, respectively. Nevertheless, we should note that the expected crystal structure and crystallinity of these ultrathin samples were confirmed by XRD, and are shown in the Figure S5. By contrast, we should note that the 'ultra-small' metal ferrites prepared at the standard reaction temperature $\left(190^{\circ} \mathrm{C}\right)$ in the presence of APTES (Figure S1B-D and S6) are amorphous and that the average size of these spherical particles (i.e. the Co3 sample) is $\sim 2.6 \mathrm{~nm}$ (Figure S6A). For the sake of brevity, all of the characterization data associated with amorphous samples have been placed in the Supplementary Information section.

Moreover, the crystallinity of our ultra-small nanoparticles were further confirmed by HRTEM and SAED patterns (Figure 4C-D and respective insets). HRTEM images in particular revealed interlayer spacings, corresponding to the expected lattice parameters associated with the spinel cubic structure of $\mathrm{Zn}$ and $\mathrm{Ni}$ ferrites, respectively. Interplanar spacings were estimated to be $\sim 0.253 \mathrm{~nm}$ and $0.249 \mathrm{~nm}$, respectively, corresponding to the (211) planes. In addition, the associated SAED patterns shown in the insets could be indexed to the reflection of a pure, crystalline spinel cubic structure.

Our ferrite nanoparticles were also analyzed by using energy-dispersive X-ray spectroscopy (EDS) to determine elemental composition. In all cases, we observed the expected peaks for ' $\mathrm{M}$ ', $\mathrm{Fe}$, and $\mathrm{O}$ as well as for $\mathrm{Si}$, the last of which could be attributed to the underlying silicon wafer substrate. Moreover, we also employed Fourier transform infrared spectroscopy and UV-visible spectroscopy to confirm the presence, most notably of Fe-Orelated signals. All of these data are described in more detail in the Supporting Information section (Figure S7). 


\section{Magnetic Characterization of Ferrites with SQUID and Mössbauer spectroscopy}

We have systematically probed a number of our ferrite nanoparticles by magnetic characterization via SQUID magnetization measurements and Mössbauer spectroscopic analysis in an effort to correlate with the particle size and composition.

\section{A1. Magnetization Analysis of $\mathrm{CoFe}_{2} \underline{O}_{4}$ nanoparticles.}

First, we explored the series of $\mathrm{CoFe}_{2} \mathrm{O}_{4}$ nanoparticles. The $\mathrm{M}$ versus $\mathrm{H}$ curves at $5 \mathrm{~K}$ (Figure 5A) and $300 \mathrm{~K}$ (Figure 5B) indicated that particles of different sizes exhibit distinctive hysteresis loops. However, the corresponding zero field-cooled (ZFC) and field-cooled (FC) magnetization curves, shown in Figure 5C, are dominated by interparticle magnetic interactions, as evinced by the rather flat FC magnetization curves. What is observed is the collective magnetic behavior of the particle ensemble, a spin-glass-like magnetic state. Bare nanoparticles interact magnetically via two mechanisms, namely (a) exchange interactions across grain boundaries and (b) dipole-dipole interactions. The former are short-ranged and can be readily eliminated by encapsulating each particle within a thin layer of surfactant. The latter, however, are longer-ranged and are present in all types of nanoparticle assemblies. In fact, they become negligible only at high magnetic dilution, i.e., at large interparticle distances and/or small particle moments. ${ }^{55-58}$ The bare $23.5 \mathrm{~nm}$ particles interact strongly through both mechanisms, whereas the surfactant-coated $14.9 \mathrm{~nm}$ diameter particles primarily interact via dipole-dipole magnetic interactions. These interactions prevent superparamagnetic relaxation and result in a collective magnetically blocked state at and below room temperature.

In the Supplementary Information (SI) section, we present additional data for 'ultrasmall' ferrite nanoparticles possessing an average diameter of $\leq 3 \mathrm{~nm}$. For the $\sim 2.6 \mathrm{~nm}$ average diameter $\mathrm{CoFe}_{2} \mathrm{O}_{4}$ surfactant-encapsulated nanoparticles investigated, the total particle moment or "macrospin" is drastically diminished, as indicated by the almost 8-fold reduction in saturation magnetization, observed at room temperature as compared with the larger particles (Table S2 and Table 2). This significantly reduces the strength of the 
interparticle dipole-dipole interaction, thereby allowing for single-particle spin reversals and the detection of superparamagnetic relaxation of individual particles. Hysteresis loops and $\mathrm{ZFC} / \mathrm{FC}$ curves are characteristically different as compared with those of the larger cobalt ferrite particles (Figure S8). The relatively sharp maximum in the ZFC magnetization curve and the $1 / T$-like increase of the magnetization as $T \rightarrow 0$ (Figure S8C) are consistent with superparamagnetism. The maximum in the ZFC magnetization curve determines the superparamagnetic blocking temperature, $T_{\mathrm{B}}$, to be about $45 \mathrm{~K}$, above which superparamagnetic behavior is expected. Thus, in this case, we observe an over-all behavior, which is consistent with the presence of magnetically non-interacting or weakly interacting magnetic nanoparticles.

It is important to note that in most cases, the magnetization of the samples did not completely saturate, even in the largest magnetic field of $5 \mathrm{~T}$ applied. This is a telltale sign of nanoparticle magnetism, attributed to spin canting at the particle surface, ${ }^{59}$ meaning that the surface spins are not perfectly aligned with the core, but tilt away from the particle's anisotropy axis. This effect is very pronounced in the case of sample Co3, measuring $2.6 \mathrm{~nm}$ in average diameter, respectively, which exhibits the most pronounced lack of magnetic saturation (Figure S8). Nonetheless, herein, the $M_{\mathrm{S}}$ has been recorded as the value of the magnetization observed at the maximum applied magnetic field of $5 \mathrm{~T}$ (Table S2).

The largest $23.5 \mathrm{~nm}$-sized particles analyzed (sample Co1) evince clear expected ferromagnetic behavior, as shown by the hysteresis loops obtained at $5 \mathrm{~K}$ and $300 \mathrm{~K}$, since the $\mathrm{ZFC} / \mathrm{FC}$ curve shows that the magnetization is blocked above $300 \mathrm{~K}$. The saturation magnetization $\left(M_{\mathrm{S}}\right)$ and coercivity $\left(H_{\mathrm{C}}\right)$ at $300 \mathrm{~K}$ are $74.5 \mathrm{emu} \mathrm{g}^{-1}$ and $849 \mathrm{Oe}$, respectively. The $M_{\mathrm{S}}$ is close to the reported value of $80.8 \mathrm{emu} \mathrm{g}^{-1}$ for bulk $\mathrm{CoFe}_{2} \mathrm{O}_{4},{ }^{60}$ which is expected, given the relatively large particle size. At $5 \mathrm{~K}$, the $M_{\mathrm{S}}$ value increases to $85.3 \mathrm{emu} \mathrm{g}^{-1}$ and the coercivity dramatically increases to 11842 Oe, consistent with previous studies. ${ }^{2,40}$ The observed increase/decrease in $M_{\mathrm{S}}$ for larger/smaller particles is likely due to the particles' 
internal spin structure, which results in the formation of a magnetic core-shell architecture, consisting of a spin-ordered core and spin-disordered shell, due to either spin canting or surface anisotropy. ${ }^{40,61}$

The $14.9 \mathrm{~nm}$ diameter particles (sample Co2) displayed a highly constricted hysteresis loop, as evinced by the presence of a sharp step at $5 \mathrm{~K}$ and $300 \mathrm{~K}$ at low applied field (insets to Figures 5A and B). These observations are consistent with some prior reports in the literature $^{28,30,40}$ and can be potentially attributed to different phenomena. First, during the synthetic process, the $\mathrm{Co}^{2+}$ ions and/or cobalt vacancies can be aligned in a preferential direction, thereby resulting in a highly anisotropic system which produces a net magnetic moment as a result of the aligned spins. Further evidence for a more magnetically anisotropic system is displayed in the higher remanence ratios $\left(M_{\mathrm{R}} / M_{\mathrm{S}}\right)$ of the cobalt ferrite nanoparticles (Table 2). Alternatively, this can also be ascribed to competition between different spin ordering structures, ${ }^{62}$ such as spin canting effects ${ }^{59}$ and/or a random distribution of cations between the (A) and [B] subsites, as aging experiments on $\mathrm{CoFe}_{2} \mathrm{O}_{4}$ have shown. ${ }^{63}$

Nanostructures are known to trap non-equilibrium states; a lack of cation order within the lattice will fail to establish a clearly preferred anisotropy axis. In this case, under a weak applied field, intrinsic random neighbor spin-spin interactions could trigger the production of a spin disordered state with a sharply reduced magnetization and a constricted hysteresis loop. Therefore, in order to assist in differentiating amongst these various plausible structural scenarios, we have employed Mössbauer spectroscopy to probe the nature of metal cation occupancies in these systems.

\section{A2. Mössbauer analysis of $\mathrm{MFe}_{2} \underline{O}_{4} \underline{\text { nanoparticles. }}$}

The room temperature (RT) Mössbauer spectra for samples Co1 and Co2 are shown in Figure 5D. They exhibit six-line absorption spectra, indicative of static magnetic order. They were fit to a superposition of two poorly-resolved iron magnetic sub-sites corresponding to 
$\mathrm{Fe}^{3+}$ at the tetrahedral $\mathrm{Fe}(\mathrm{A})$ and octahedral $\mathrm{Fe}[\mathrm{B}]$ sites, including distributions of hyperfine fields. The ultra-small $2.6 \mathrm{~nm}$ nanoparticles (sample Co3), on the other hand, exhibited a collapsed quadrupolar spectrum at RT (Figure S9, top spectrum), consistent with the presence of superparamagnetism. This spectrum was fit to the superposition of two poorly resolved quadrupolar sub-sites (Table S3), corresponding to the $\mathrm{Fe}(\mathrm{A})$ and $\mathrm{Fe}[\mathrm{B}]$ spinel crystallographic sites, with the larger quadrupole of $\Delta \mathrm{E}_{\mathrm{Q} 1}=0.94 \pm 0.05 \mathrm{~mm} / \mathrm{s}$, associated with the tetrahedral (A) site, and the smaller quadrupole of $\Delta \mathrm{E}_{\mathrm{Q} 2}=0.53 \pm 0.05 \mathrm{~mm} / \mathrm{s}$, correlated with the more symmetric octahedral [B] site.

Table 3 summarizes some of the fitted hyperfine parameters values obtained for the various ferrite nanosystems studied. The hyperfine field distributions experienced by the iron nucleus at a particular site is sensitive to the number of $\mathrm{M}^{2+}$ nearest neighbors, which gives rise to the observed field distributions. For the case of $\mathrm{CoFe}_{2} \mathrm{O}_{4}$, the average magnetic fields at both $\mathrm{Fe}(\mathrm{A})$ and $\mathrm{Fe}[\mathrm{B}]$ sites are considerably reduced as compared with the corresponding bulk values of $\mathrm{B}_{\mathrm{HF}}(\mathrm{A})=511.5 \mathrm{kOe}$ and $\mathrm{B}_{\mathrm{HF}}[\mathrm{B}]=550 \mathrm{kOe}$, as a result of finite size effects. ${ }^{64} \mathrm{We}$ note that depending on particle size, the spectra can exhibit either quadrupole doublets (fast spin relaxation) or magnetic sextets (slow spin relaxation). For example, in the case of the 7.4 $\mathrm{nm} \mathrm{Ni} 2$ ferrite sample, Table 3 indicates that satisfactory fits necessitated the inclusion of small contributions of dynamic, intermediate spin relaxation sub-spectra associated with sites (A) and [B] with much reduced hyperfine magnetic fields.

It is also important to highlight the difference in the DOIs, which measure the relative $\mathrm{Fe}^{3+}$ site occupancy in tetrahedral and octahedral sites, derived from the ratio of the spectral absorption areas $\mathrm{Fe}(\mathrm{A}) / \mathrm{Fe}[\mathrm{B}]$ in the Mössbauer spectra. In a normal spinel structure, $\mathrm{M}^{2+}$ ions should all reside at the tetrahedral (A) site, whereas for an inverse spinel structure, these ions are localized at the octahedral [B] site. Indeed, the site occupancies can significantly affect the magnetization values of the sub-lattices and, therefore, the overall magnetization of the sample. Previous reports on both nanosized and bulk $\mathrm{CoFe}_{2} \mathrm{O}_{4}{ }^{64,65}$ have suggested a range of 
different degrees of inversion. For example, bulk Co ferrite thermally treated at $1200^{\circ} \mathrm{C}$ yielded a $\mathrm{Fe}(\mathrm{A}) / \mathrm{Fe}[\mathrm{B}]$ value of $0.92 \pm 0.04$ for slowly cooled samples but an analogous number of $0.65 \pm 0.03$ for rapidly quenched samples. ${ }^{65}$

For nanosized powders ${ }^{64}$ without any thermal treatment, the ratio of $\mathrm{Fe}(\mathrm{A}) / \mathrm{Fe}[\mathrm{B}]$ was found to be $0.73 \pm 0.07$. Our $\mathrm{CoFe}_{2} \mathrm{O}_{4}$ nanostructures exhibited degrees of inversion of $0.39 \pm$ $0.09,0.45 \pm 0.10$, and $0.37 \pm 0.09$ for the $2.6 \mathrm{~nm}$ (sample Co3), $14.9 \mathrm{~nm}$ (sample Co2), and $23.5 \mathrm{~nm}$ particles (sample Co1), respectively. These results reinforce observations in the literature that the degree of inversion (DOI) is affected by the synthetic protocol, processing techniques, and processing treatments. More importantly, our analysis shows that both magnetization and the DOI values are highly sensitive to nanoparticle size. Specifically, the increase in the DOI numbers with decreasing particle size implies that more $\mathrm{Co}^{2+}$ ions may be occupying more of the $[\mathrm{B}]$ sites, as the size is reduced.

\section{A3. SQUID and Mössbauer analysis of Amorphous Ultra-small $2.6 \mathrm{~nm} \mathrm{CoFe} 2 \underline{O}_{4} \underline{\text { nanoparticles }}$.}

Of importance for properly understanding the effect of size, the observation of full superparamagnetic behavior in sample Co3 with a $2.6 \mathrm{~nm}$ diameter has allowed for complete micromagnetic characterization of this system. ${ }^{66}$ The superparamagnetic relaxation time (Equation 1) is modeled by the celebrated Néel relaxation process. ${ }^{67-70}$

$$
\tau_{s}=\tau_{0} e^{\frac{K V}{k T}}
$$

Here, $\tau_{s}$ gives the relaxation time of the particle's "macrospin", $\tau_{0}$ is the attempt time for spin reversals, $K$ yields the uniaxial magnetic anisotropy density, $V$ represents the volume of the particle, $k$ is Boltzmann's constant, and $T$ denotes the temperature; $K V$ provides the anisotropy energy or energy barrier for spin reversals. For cubic anisotropy, as is the case for magnetite as well as the spinel ferrites, the uniaxial anisotropy constant $K$ is replaced by $K_{1} / 4,{ }^{71-73}$ where $K_{1}$ is the first coefficient of magnetocrystalline anisotropy. Unlike $T_{\mathrm{B}}$, which is technique 
dependent, $K$ and the pre-exponential factor $\tau_{0}$ represent intrinsic physical properties of the nanoparticles. Determination of the blocking temperature $T_{\mathrm{B}}$ using two magnetic characterization techniques with different characteristic measuring time windows, $\tau_{m}$, permits for the corresponding analysis of the values of $\tau_{0}$ and $K$. Hence, we have combined (i) SQUID magnetization measurements, shown in Figure S8, with $\tau_{m} \sim 10-100 \mathrm{~s}$, and (ii) temperature dependent Mössbauer spectroscopic studies, shown in Figure S9, with $\tau_{m} \sim 10^{-8} \mathrm{~s}$, in order to obtain a complete micromagnetic characterization of this system. As discussed earlier (Figure S8 and S9), Co3 exhibits no evidence for the presence of interparticle interactions, either in its Mössbauer or SQUID behavior. This is due to the extremely small size of the particle and its lack of crystallinity; amorphous samples are expected to exhibit internal spin disorder, which results in a concomitant small "macrospin", thereby rendering interparticle dipole-dipole interactions negligible.

Figure S9 and accompanying Table S4 provide the Mössbauer spectral profile over the temperature range of $4.2 \leq \mathrm{T} \leq 300 \mathrm{~K}$. A blocking temperature of $T_{\mathrm{B}}$ (Mössbauer) $\sim 75 \mathrm{~K}$ is observed, while the maximum at $T_{\mathrm{B}}$ (SQUID) $\sim 45 \mathrm{~K}$ in Figure $\mathrm{S} 8 \mathrm{C}$ gives the average blocking temperature for magnetization measurements. As the coexistence of doublets and sextets in the observed Mössbauer spectra extends over a large temperature range, the blocking temperature is determined where the area of the doublets and sextets are equal; this designates the average blocking temperature for the ensemble of the nanoparticles in the sample. Information about the average blocking temperature and average nanoparticle size has been used in Equation (1) to obtain $K=2.5 \cdot 10^{4} \mathrm{~J} / \mathrm{m}^{3}$ and $\tau_{0}=0.5 \cdot 10^{-9} \mathrm{~s}$. Bulk $\mathrm{CoFe}_{2} \mathrm{O}_{4}$, as with all spinels, possesses cubic magnetic anisotropy, ${ }^{61-63}$ with its first coefficient of magnetocrystalline anisotropy, $K_{1}$, in the range of $(1.8-3.0) \cdot 10^{5} \mathrm{~J} / \mathrm{m}^{3}$, while $\tau_{0}$ is of the order of a timescale of $10^{-9} \mathrm{~s}$, usually assumed for superparamagnetic nanoparticles. We note that the value of $K$ obtained is an order of magnitude smaller than that of the magnetocrystalline 
anisotropy of the bulk, in contradiction to prior reports of increased anisotropy of up to two orders of magnitude for nanoparticles, due to shape, surface, and strain anisotropy contributions. ${ }^{74,75}$ Moreover, the $K$ value is consistent with use in the PDF studies presented in Section III.

Nevertheless, we believe that this is not necessarily a surprising result, since at a particle diameter of only $2.6 \mathrm{~nm}$, these systems are well below the critical magnetic characteristic exchange length scale for collinear magnetic sublattice formation in $\mathrm{CoFe}_{2} \mathrm{O}_{4}$, i.e. $\ell_{e x}=5.2 \mathrm{~nm} .{ }^{76}$ The critical exchange length is given by $\ell_{e x}=\sqrt{\frac{A}{\mu_{0} M_{s}}}$, where ' $A$ ', represents the Co ferrite lattice exchange stiffness, which depends upon the strength of the superexchange interactions between neighboring spins and the strength of the magnetocrystalline anisotropy. ${ }^{77}$ Therefore, size matters in as much as spin canting and disorder effects permeate throughout the entire volume of this ultra-small Co particle rather than being confined at the surface. This assertion explains the much reduced $M_{\mathrm{S}}$ for this system (Table S2).

\section{B. Magnetic Behavior of nanoscale $\mathrm{Mg}, \mathrm{Zn}, \mathrm{Cu}$, and Ni ferrites.}

The magnetic characterization data for $\mathrm{MgFe}_{2} \mathrm{O}_{4}$ (samples $\mathrm{Mg} 1$ ), $\mathrm{ZnFe}_{2} \mathrm{O}_{4}$ (sample $\mathrm{Zn1}$ ), $\mathrm{CuFe}_{2} \mathrm{O}_{4}$ (sample $\mathrm{Cu} 1$ ), and $\mathrm{NiFe}_{2} \mathrm{O}_{4}$ (samples $\mathrm{Ni1}$ and $\mathrm{Ni3}$ ) are comparatively shown in Figure 6. Details of size and composition effects are explicitly discussed below in Section $\mathrm{B} 1$ for $\mathrm{Mg}$ and $\mathrm{Zn}$ ferrites and Section B2 for the corresponding $\mathrm{Cu}$ and Ni ferrites.

Generally speaking, except for the ultra-small $3.0 \mathrm{~nm} \mathrm{ZnFe}_{2} \mathrm{O}_{4}$ nanoparticles (sample $\mathrm{Zn} 2$ ), hysteresis was observed at $5 \mathrm{~K}$, which is characteristic of ferromagnetic behavior. Furthermore, with the exception of $\mathrm{Zn} 1$ and $\mathrm{Zn} 2$, the ZFC-FC curves of these samples are dominated by the presence of interparticle magnetic interactions, as evinced by the broad maxima of the ZFC curves and the 'flattened out' features of the ZF-curve below $\mathrm{T}_{\max }$. The 
temperature at which the maximum occurs $\left(T_{\max }\right)$ marks the spin-glass transition temperature in the collective magnetic behavior of the nanoparticle assemblies. The observed $T_{\max }$ values (Table 2) for samples Mg1 (32.9 nm), Cu1 (13.3 nm), Ni1 (87.3 nm), and Ni3 (3.1 nm) were found to be $>300 \mathrm{~K}, 165 \mathrm{~K},>300 \mathrm{~K}$, and $29 \mathrm{~K}$, respectively. Not surprisingly, the relatively large $\mathrm{MgFe}_{2} \mathrm{O}_{4}$ nanoparticles still displayed a small degree of hysteresis at $300 \mathrm{~K}$.

Interestingly, although the magnetic spin-freezing temperature was determined to be $165 \mathrm{~K}$, a small hysteresis loop was still present at $300 \mathrm{~K}$ for the $13.3 \mathrm{~nm} \mathrm{Cu}$ ferrite nanoparticles as well as for the $7.4 \mathrm{~nm} \mathrm{NiFe} \mathrm{O}_{4}$ nanoparticles, a situation which might have been caused either by the trapping field in the superconducting magnets in the SQUID magnetometer or by the presence of some larger or agglomerated nanoparticles in the distribution. Therefore, in some instances, determining $T_{\mathrm{B}}$ from the maximum of the ZFC curve is not necessarily accurate, a finding which has been demonstrated previously. ${ }^{4}$ As for the corresponding as-prepared ultrasmall amorphous samples, all of the relevant magnetic and Mössbauer data tables and curves are further discussed in the Supplementary Information section (i.e. Tables S2, S3, and S4, as well as Figures S8, S9, and S10).

\section{B1. SQUID and Mössbauer analysis of nanoscale $M g$ and $\mathrm{Zn}$ ferrites}

Sample Zn1 (Figure 6D) shows a relatively sharp maximum at the depressed temperatures of $22 \mathrm{~K}$. This maximum is consistent with superparamagnetic blocking temperatures $\left(T_{\mathrm{B}}\right)$. However, the observation that the $\mathrm{FC}$ curve does not keep increasing at temperatures below $T_{\mathrm{B}}$ is inconsistent with simple superparamagnetic behavior and may indicate the onset of antiferromagnetic interactions. In the bulk, $\mathrm{ZnFe}_{2} \mathrm{O}_{4}$ is a normal spinel with the $\mathrm{Zn}^{2+}$ ions occupying exclusively tetrahedral (A) sites; it is paramagnetic down to 10 $\mathrm{K}$, where it undergoes a paramagnetic-to-antiferromagnetic phase transition. In the nanostructured state, however, changes in the degree of inversion have been reported, resulting in competing ferromagnetic and antiferromagnetic interactions and the production of 
superparamagnetic nanoparticles with blocking temperatures well above $10 \mathrm{~K}$ but below room temperature. ${ }^{27}$ Our $3.0 \mathrm{~nm} \mathrm{ZnFe}_{2} \mathrm{O}_{4}$ particles exhibited a degree of inversion of 0.59 , with $33 \%$ of $\mathrm{Fe}^{3+}$ ions occupying tetrahedral (A) sites, as determined from Mössbauer spectroscopy (Table 3).

In the case of the $\mathrm{Mg} 1$ sample, the presence of a magnetic iron signature in the Mössbauer spectra of a relatively large isomer shift (i.e. $0.65 \mathrm{~mm} \mathrm{~s}^{-1}$ at $300 \mathrm{~K}$, Table 4) points to the partial reduction of $\mathrm{Fe}^{3+}$ to $\mathrm{Fe}^{2+}$, accompanied by the partial oxidation of $\mathrm{Mg}^{2+}$ to $\mathrm{Mg}^{3+}$, with concomitant changes in the intrinsic magnetic interactions in this system. This finding may be primarily due to the complicating presence of magnetite, $\mathrm{Fe}_{3} \mathrm{O}_{4}$, within the $\mathrm{Mg} 1$ sample itself (Figure 1), as it appears to comprise $\sim 44 \%$ of the magnetic sub-spectrum. Nevertheless, this plausible scenario cannot be readily confirmed through XRD, because the positions of the diffraction peaks for both spinel structures, i.e. $\mathrm{Fe}_{3} \mathrm{O}_{4}$ and $\mathrm{MgFe}_{2} \mathrm{O}_{4}$, are very similar. In the magnetic data, the presence of hysteresis gives rise to a higher $M_{\mathrm{S}}$ value at 300 $\mathrm{K}$ of $34.0 \mathrm{emu}^{-1}$ with a small coercivity of 78 Oe. The observation of high saturation magnetization coupled with low coercivities is indicative of ferrimagnetic behavior, ${ }^{78}$ and that fact is consistent with the known behavior of nanoscale $\mathrm{Mg}$ ferrite. ${ }^{79,80}$

We should note that the DOI of the Mg1 sample is higher than 1, based upon fitting of the Mössbauer spectra (c.f. Table 3). For highly crystalline spinel bulk materials, the maximum DOI expected is 1 . In the presence of disorder and/or defects in the nanocrystalline state, DOI could conceivably increase above 1 . This may be due to the fact that surface sites, which can dramatically increase in proportion relative to the bulk at the nanoscale, will behave more like (A) sites on account of the distorted/reduced coordination at the surface, thereby explaining the Ni1 and Ni2 data, shown in Table 3.

The $7.8 \mathrm{~nm} \mathrm{Zn}$ ferrite nanoparticles (sample $\mathrm{Zn} 1$ ) possess $M_{\mathrm{S}}$ values of $54.5 \mathrm{emu}^{-1}$ and $15.2 \mathrm{emu} \mathrm{g}^{-1}$ at $5 \mathrm{~K}$ and $300 \mathrm{~K}$, respectively (Figure 6C). At different temperatures, wide ranges of saturation values have been reported from $\sim 20-89 \mathrm{emu} \mathrm{g}^{-1}$ at $5 \mathrm{~K}$ and $10-75 \mathrm{emu}$ 
$\mathrm{g}^{-1}$ at $300 \mathrm{~K}$ for $\mathrm{ZnFe}_{2} \mathrm{O}_{4}$ nanoparticles. ${ }^{27}$ Our data fall within these ranges at both temperatures, representative of a $\mathrm{Fe}^{3+}$ cation redistribution between the tetrahedral (A) and octahedral [B] sites ${ }^{81}$ which we had also already observed for the Mg ferrite system.

Moreover, the $7.8 \mathrm{~nm}$ particles exhibit a flatter $\mathrm{M}$ versus $\mathrm{H}$ curve at $300 \mathrm{~K}$, consistent with the presence of a paramagnetic material, in which $M_{\mathrm{S}}$ is $15.2 \mathrm{emu} \mathrm{g}^{-1}$.

This paramagnetic behavior of the $7.8 \mathrm{~nm} \mathrm{ZnFe}_{2} \mathrm{O}_{4}$ nanoparticles was confirmed subsequently by RT Mössbauer spectroscopy. While this sample (Zn1) also exhibited a collapsed quadrupole spectrum as did the $2.6 \mathrm{~nm} \mathrm{CoFe}{ }_{2} \mathrm{O}_{4}$ sample, the origin is likely to be paramagnetic. The $\mathrm{Zn}$ particles exhibited smaller quadrupole splitting along with narrower line widths by comparison (Table 3). This observation is not surprising, given that the Néel temperature $\left(T_{\mathrm{N}}\right)$ of bulk $\mathrm{ZnFe}_{2} \mathrm{O}_{4}$ is $10 \mathrm{~K} .{ }^{82}$ Even though cation redistribution at the nanoscale is known to produce ferrimagnetism ${ }^{27}$ as previously discussed, the sample is likely to be paramagnetic at room temperature.

As expected, the ultra-small $\mathrm{ZnFe}_{2} \mathrm{O}_{4}$ nanoparticles (sample $\mathrm{Zn} 2$ ) exhibited Mössbauer quadrupole behavior. Spectral fits to the superposition of two poorly resolved quadrupole doublets, corresponding to (A) and [B] crystallographic sites, indicated that the iron coordination within the ultra-small $\mathrm{ZnFe}_{2} \mathrm{O}_{4}$ nanoparticles is severely distorted away from perfect tetrahedral and octahedral coordination symmetry. The value of the quadrupole splitting for $\mathrm{Fe}(\mathrm{A})$ sites was $0.82 \mathrm{~mm} \mathrm{~s}^{-1}$ and that for the $\mathrm{Fe}[\mathrm{B}]$ sites was $0.44 \mathrm{~mm} \mathrm{~s}^{-1}$. The corresponding values for the $7.8 \mathrm{~nm} \mathrm{Zn}$ ferrite particles were $0.61 \mathrm{~mm} \mathrm{~s}^{-1}$ and $0.35 \mathrm{~mm} \mathrm{~s}^{-1}$, respectively (Table 3). Their degree of inversion also differed; sample Zn1 possessed a degree of inversion of 0.85 , while for $\mathrm{Zn} 2$, it was reduced to 0.59 . To further emphasize size effects, cobalt ferrite particles also displayed a reduction in their degree of inversion with decreasing particle size, with the $2.6 \mathrm{~nm} \mathrm{Co} 3$ and $14.9 \mathrm{Co} 2$ particles, yielding DOI values of 0.39 and 0.45. Interestingly, the amorphous sample, i.e. the $2.6 \mathrm{~nm} \mathrm{Co3}$ particles, also evinced a similar temperature-dependent trend, yielding DOI values of 0.39 and 0.51 , when measured at room 
temperature (Table S3) and at $4 \mathrm{~K}$ (Table S4), respectively. This observation either may potentially indicate cation redistribution or may arise from a differential temperature dependence of the recoil free fraction at (A) and [B] sites, or both.

\section{B2. SQUID and Mössbauer analysis of nanoscale Cu and Ni ferrites}

The $\mathrm{M}$ versus $\mathrm{H}$ curves of the copper ferrite system (sample Cu1) are quite distinctive from that of the other ferrite nanoparticle systems (Figure 6E, F). Most notably, at $5 \mathrm{~K}$ and $300 \mathrm{~K}$, the nanoparticles exhibited very low $M_{\mathrm{S}}$ values of $4.5 \mathrm{emu} \mathrm{g}^{-1}$ and $5.5 \mathrm{emu} \mathrm{g}^{-1}$, respectively. Hence, we can assume that the magnetic saturation of the sample is largely unaffected by the change in temperature. However, the coercivity decreases from 485 Oe to 2 Oe upon increasing the temperature from $5 \mathrm{~K}$ to $300 \mathrm{~K}$. As such, a small, irregular hysteresis is still present. While these values are significantly lower than those associated with other studies on similarly sized $\mathrm{CuFe}_{2} \mathrm{O}_{4}$ nanoparticles obtained through ball milling ${ }^{83}$ and chemical methods,${ }^{84}$ relatively small values similar to ours have been reported for similarly sized $\mathrm{CuFe}_{2} \mathrm{O}_{4}$ nanoparticles obtained through a sol-gel route. ${ }^{85}$ The composition-dependent Mössbauer spectra evinced the smallest degree of inversion among all ferrite nanosystems studied with a value of 0.28 .

Interestingly, the $87.3 \mathrm{~nm}$ (sample $\mathrm{Ni} 1$ ), and $7.4 \mathrm{~nm}$ (sample $\mathrm{Ni2}$ ) $\mathrm{NiFe}_{2} \mathrm{O}_{4}$ samples exhibited rather similar magnetic behavior, regardless of their difference in size (Figure 6G, H). The large Ni1 sample displayed a hysteresis at both $5 \mathrm{~K}$ and $300 \mathrm{~K}$, with coercivities of 436 and 43 Oe, respectively, which is expected given that the spin-freezing magnetic transition was found to occur above $300 \mathrm{~K}$ (Figure $6 \mathrm{H}$ ). At $300 \mathrm{~K}$, the saturation magnetization was $46.0 \mathrm{emu} \mathrm{g}^{-1}$, which is very close to the value of $47.5 \mathrm{emu} \mathrm{g}^{-1}$ reported for bulk $\mathrm{NiFe}_{2} \mathrm{O}_{4}{ }^{86}$ It is noteworthy that for the Ni1 sample, an additional contributing factor to the broad maxima may be its relatively large size distribution (see Table 1). ${ }^{87}$ The bulk-like 
magnetic behavior of these particles is expected, given the much larger average particle size as compared with the other ferrite systems tested.

However, the ultra-small $\sim 3 \mathrm{~nm}$ Ni particles (i.e. Ni3 sample) displayed a somewhat different magnetic behavior with a measured spin-glass freezing temperature of $261 \mathrm{~K}$ (Table 2) and we also noted the presence of a slight hysteresis at $300 \mathrm{~K}$ with a small coercivity of 17 Oe. Interestingly, above $2 \mathrm{~K}$, the increase in field magnitude with temperature of the ZFC curve attained a maximum, observed at approximately $T_{B}=29 \mathrm{~K}$, a phenomenon which could be ascribed to the presence of superparamagnetic crystallites. These ultra-small particles also evinced relatively low $M_{\mathrm{S}}$ values (i.e. $12.1 \mathrm{emu} \mathrm{g}^{-1}$ ), as opposed to bulk $\mathrm{NiFe}_{2} \mathrm{O}_{4}$ (i.e. $\mathrm{M}_{S}=55$ emu/g) ${ }^{88}$ This observation overall may be potentially attributed to superparamagnetism at the nanoscale. By contrast, at $300 \mathrm{~K}$, the Ni ferrite systems (i.e. $7.4 \mathrm{~nm}$ and $87.3 \mathrm{~nm}$ ) behaved like soft magnetic materials with high saturation values and low coercivities. This behavior is also consistent with ferrimagnetic behavior.

The observed behavior of the degree of inversion of the Ni samples, as reported in Table 3, is in need of further explanation. In the bulk, $\mathrm{NiFe}_{2} \mathrm{O}_{4}$ is known to be an inverse spinel with $\mathrm{Ni}^{2+}$ ions residing on [B] sites and $\mathrm{Fe}^{3+}$ ions residing in (A) and [B] sites. Thus, in the absence of defects, bulk $\mathrm{NiFe}_{2} \mathrm{O}_{4}$ should exhibit a degree of inversion of 1 . Both Ni1 (87.3 $\mathrm{nm})$ and Ni2 (7.4 $\mathrm{nm})$ exhibit degree of inversions larger than 1, which can be explained by the presence of surface sites that behave more like (A) sites, due to the decrease of iron coordination at the surface. One would have expected a monotonic increase in the degree of inversion as the particle size becomes even smaller, namely with our Ni3 ( $3 \mathrm{~nm})$ samples, as the surface to bulk ratio increases with decreasing particle size. By contrast, the degree of inversion for $\mathrm{Ni3}$ decreases to 0.74 . This observation is consistent with previous reports that ultra-small particle sized nanocrystalline $\mathrm{NiFe}_{2} \mathrm{O}_{4}$ becomes a mixed spinel, with some $\mathrm{Ni}^{2+}$ ions forced to occupy (A) sites. ${ }^{89}$ This will decrease the $\mathrm{Fe}^{3+}$ occupancy of (A) sites, thereby producing the observed reduction in the degree of inversion in $\mathrm{Ni3}$. 


\section{Coupling Mössbauer and Magnetic Data of Ferrites with Related PDF Analysis.}

The premise of using the pair distribution function (PDF) is that materials are made of multiple elements, have large unit cells, and are often low dimensional or incommensurate structures. ${ }^{90}$ Increasingly also, they have aperiodic disorder with some aspect of the structure that is clearly different from the average crystal structure. In the case of nanoparticles, the very concept of a crystal is invalid, as the approximation of infinite periodicity is no longer a good one. Hence, the value added of a 'total scattering' experiment generating PDF data over a conventional powder diffraction analysis is the inclusion of diffuse scattering as well as Bragg peak intensities in the analysis, and the wide range of $Q$ (the magnitude of the scattering vector) over which data are measured. 'Total scattering' data can be analyzed by fitting models directly in reciprocal-space. ${ }^{91}$

Hence, in light of the extensive magnetization data collected and interpreted, we attempted to further understand and refine the crystal structure of the ferrite samples using PDF analysis, which has already been previously applied to systems ranging from ultra-small CdSe quantum dots, with diameters of $2 \mathrm{~nm}$ to $4 \mathrm{~nm}$, as well as to existing metal oxide systems, such as $\mathrm{YMnO}_{3}$ particles, measuring from $10 \mathrm{~nm}$ to $467 \mathrm{~nm} .{ }^{53,92}$ However, though $\mathrm{MgFe}_{2} \mathrm{O}_{4}$ and $\mathrm{CoFe}_{2} \mathrm{O}_{4}$ have been analyzed in the past ${ }^{93-95}$ using PDF, the size regime of these specific particles was nominally $6 \mathrm{~nm}$ or higher, and these ferrite materials conserved their cubic spinel structure. Herein, we are currently the first to report on a PDF analysis of a range of variously-sized nanoparticulate metal oxide samples.

For illustrative purposes, $\mathrm{Co}, \mathrm{Zn}$, and $\mathrm{Ni}$ ferrites with various size regimes were rigorously analyzed (Table 4). The PDFs of all the ferrite nanoparticles are plotted in the $r$ range up to $40 \AA$ in Figure 7 . The PDF peaks for Co1, Co2, Ni1, and Ni2 samples are still strong at $40 \AA$ where the end point is designated, indicating well-ordered local structures in these materials. By contrast, the PDF peak intensities in Zn1, Zn2, and Ni3 samples diminish 
more quickly with increasing $r$ values, indicating a smaller structural coherence for these samples. The PDFs with a high degree of structural coherence (i.e. Co1, Co2, Ni1, and Ni2) are very similar, suggesting these samples share the same structure with each other. However, apart from the reduced size of the particles, PDF patterns of Ni3, Zn1, and $\mathrm{Zn} 2$ are similar to the well-ordered Ni and cobalt PDFs, indicating that the underlying structure is the same.

In order to extract more quantitative structural information, we carried out PDF model fits, using the structural model for the cubic spinel space group $F d \overline{3} m$ (No. 227), and calculated reasonable agreement values with the experimental Synchrotron Light Source XRD and electron microscopy diffraction data $\left(R_{w}\right)$ as shown in Figure 8 , with the fit results summarized in Table 4. We should note that due to similar scattering potentials of the Co and Ni samples, the DOIs (as defined in the Experimental section below) were derived from previous Mössbauer experiments (see Table 3) and were used in the model fits. By an inspection of the fits, we observe a good agreement between the measured data and the simulated models for $\mathrm{Co} 1, \mathrm{Co} 2, \mathrm{Ni} 1$, and $\mathrm{Ni} 2$ with an $R_{w}$ value of $\sim 0.1$. The structural features of these larger particles are well described by the cubic spinel model. As the particle size decreases, the fits become worse, being in the vicinity of $R_{w}=0.4-0.5$. This indicates that there is some 'unfit' component in the signal which may originate from ligand effects, for example, of the small nanoparticles. In the ePDFs of Zn2 and Ni3, there is also a residual signal from the carbon grid that supported the nanoparticles, which was not completely subtracted during the background correction. Nonetheless, it is clear that the best-fit PDFs from the models explain the structure well, with calculated peaks lining up well with the measured PDF. We therefore trust the refinements quite well and the poor $R_{w}$ can be attributed to signals from impurity components as opposed to a poor fit to the nanoparticle structures. The refined parameters in Table 4 show good agreement between the ultra-small and the larger nanoparticles. 
The range of signal in the PDF, which indicates the range of structural coherence, is comparable to the physical size of the particles observed in the TEM. Both of these observations suggest that the degree of structural order in the ultra-small nanoparticles is actually rather small. In effect, they appear to be very small chunks of well-defined spinel material. Notably, all the samples, especially the Ni and Zn, exhibit a very high degree of inversion between the tetrahedral and octahedral sites. There appears to be no clear structural explanation for the different magnetic response of the ultra-small nanoparticles. It is interesting to note, however, that this ultra-small particle size range coincides with the characteristic magnetic exchange length scale for magnetic sublattice formation as earlier discussed in light of the Mössbauer analysis of $\mathrm{Co3}$, and the structural disorder therefore may have a significant effect upon the magnetic properties at the smallest nanoparticle size-scale. The data table of PDF fits for the rest of the ferrite samples analyzed can be found in the Supporting Information section (Table S5).

\section{Catalysis of Ferrites.}

\section{(i). Photocatalytic Activity}

It has been previously demonstrated that ferrite systems, specifically cobalt and zinc, can be utilized as photocatalysts. Generally, ferrites are utilized in composite systems, since these systems alone are not effective at separating the electron-hole pairs generated by the irradiation process (Equation 2). Hence, a second component, such as graphene, is often present, which is believed to not only provide a pathway for electron transport and prevent undesired recombination but also react with dissolved oxygen in order to produce superoxide anion radicals. ${ }^{18}$

$$
\begin{aligned}
& \mathrm{MFe}_{2} \mathrm{O}_{4}+h v \rightarrow \mathrm{MFe}_{2} \mathrm{O}_{4}\left(\mathrm{~h}^{+}+\mathrm{e}^{-}\right) \\
& \mathrm{MFe}_{2} \mathrm{O}_{4}\left(\mathrm{~h}^{+}\right)+\mathrm{OH}^{-} \rightarrow \mathrm{MFe}_{2} \mathrm{O}_{4}+{ }^{\circ} \mathrm{OH}
\end{aligned}
$$


Therefore, the holes, remaining on the ferrite, can react with adsorbed water in order to form hydroxyl radical species (Equation 3). Collectively, these active species, including holes and radicals generated by the electron acceptor, can subsequently oxidize the various tested dyes.

In this work, we have probed relatively simple reactions, involving the degradation of methylene blue (MB) under UV irradiation conditions in the presence of our as-synthesized $\mathrm{MFe}_{2} \mathrm{O}_{4}$ nanoparticles. The photocatalytic activity was determined by monitoring the optical behavior of MB at $664 \mathrm{~nm}$, which represents the peak absorbance of the dye, as a function of time. As shown in Figure 9A, the nanoparticles (at a concentration of $0.25 \mathrm{~g} / \mathrm{L}$ ) are active as photocatalysts as compared with the control sample. Moreover, we found that their chemical composition apparently greatly influences the observed activity. Specifically, we note that our $32.6 \mathrm{~nm} \mathrm{MgFe} 2 \mathrm{O}_{4}$ (sample Mg1) nanoparticles exhibited the highest photocatalytic activity. This enhancement may be attributed to the interaction of the $\mathrm{Mg} 1$ sample with both $\mathrm{Fe}_{2} \mathrm{O}_{3}$ and $\mathrm{Fe}_{3} \mathrm{O}_{4}$ potential impurities in the sample, a process which may inhibit recombination and thereby facilitate the generation of oxidative species, such as hydroxyl radicals, relevant for methylene blue degradation. ${ }^{96,97}$ The high performance of the Mg sample is followed by that of our $7.4 \mathrm{~nm} \mathrm{NiFe} \mathrm{O}_{4}$ sample (sample Ni2), $13.3 \mathrm{~nm} \mathrm{CuFe}_{2} \mathrm{O}_{4}$ (sample Cu1), $7.8 \mathrm{~nm}$ $\mathrm{ZnFe}_{2} \mathrm{O}_{4}$ (sample $\mathrm{Zn1}$ ), and $14.9 \mathrm{~nm} \mathrm{CoFe}_{2} \mathrm{O}_{4}$ (sample Co2) nanoparticles, respectively, in order of decreasing activity.

We were able to model the raw data as a pseudo-first-order reaction (Figure 8B), with the kinetics governed by the equation $\ln \left(\mathrm{C}_{0} / \mathrm{C}_{\mathrm{t}}\right)=\mathrm{kt}$, wherein $\mathrm{C}_{0}$ is the initial concentration of $\mathrm{MB}, \mathrm{C}_{\mathrm{t}}$ is the concentration of $\mathrm{MB}$ at a given time ' $\mathrm{t}$ ', and ' $\mathrm{k}$ ' is the reaction rate constant derived from the slopes of the 'best' fit lines. From this analysis, we were able to calculate pseudo-rate constants for $\mathrm{Mg} 1, \mathrm{Ni} 2, \mathrm{Cu} 1, \mathrm{Zn} 1$, and $\mathrm{Co} 2$ of $6.97 \cdot 10^{-4}, 4.64 \cdot 10^{-4}, 4.04 \cdot 10^{-4}$, $3.05 \cdot 10^{-4}$, and $2.75 \cdot 10^{-4} \mathrm{~min}^{-1}$, respectively. It is worth noting that the $\mathrm{Mg}$ and $\mathrm{Zn}$ sample data do not necessarily follow a first-order fit, whereas the remainder of the ferrite nanoparticles essentially do (Table S6). By means of comparison, we have also plotted the data as pseudo- 
second-order fits (Figure S13 and Table S7). We have found that the fits improved slightly for both $\mathrm{Mg}$ and $\mathrm{Zn}$ series, whereas the first order fits were still better for $\mathrm{Co}, \mathrm{Ni}$, and $\mathrm{Cu}$ ferrites, respectively. Nonetheless, as noted from the linear fits of the data (Figure 9B), the calculated rate constants confirmed reasonable photocatalytic activity for our ferrite nanoparticles.

Most importantly, by comparison with the available literature, our as-prepared ferrite nanoparticles evinced higher activities at lower concentrations. Specifically, Fu et al. reported that $\mathrm{CoFe}_{2} \mathrm{O}_{4}$ nanoparticles at a concentration of $1.0 \mathrm{~g} / \mathrm{L}$ alone were photocatalytically inert under visible-light irradiation. ${ }^{18}$ Although we utilized UV-irradiation in this work, we have demonstrated that $\mathrm{CoFe}_{2} \mathrm{O}_{4}$ nanoparticles are in fact photocatalytically active, even at a lower concentration of $0.25 \mathrm{~g} / \mathrm{L}$. Similarly, in the case of $\mathrm{ZnFe}_{2} \mathrm{O}_{4}$, we demonstrated that our nanoparticles are photocatalytically active at $0.25 \mathrm{~g} / \mathrm{L}$ concentrations, although previous works have reported that $\mathrm{ZnFe}_{2} \mathrm{O}_{4}$ at a concentration of $1.0 \mathrm{~g} / \mathrm{L}$ possessed either little or no photocatalytic activity under visible-light irradiation. ${ }^{17,} 19$ To the best of our knowledge, we are the first to report upon the photocatalytic activity of $\mathrm{Mg}, \mathrm{Ni}$, and $\mathrm{Cu}$ ferrites. In fact, our data suggest that these particular ferrites may be more efficient for photocatalytic applications as opposed to either $\mathrm{Zn}$ or Co ferrites, which have been utilized to date.

The UV-visible data for these samples (Figure S7C) may also shed some light on our observations. Of the samples explored, both the $\mathrm{Ni}$ and $\mathrm{Cu}$ ferrites exhibited evident peaks in their spectra which were large and broad. The $\mathrm{Zn}$ ferrite sample also gave rise to a noticeable peak, which was much narrower than that found for either the $\mathrm{Ni}$ or $\mathrm{Cu}$ samples. Cobalt and iron maintained generally low absorbances over a broad absorption range. Hence, the Ni and $\mathrm{Cu}$ samples can apparently absorb more light than the remaining ferrites, and therefore possess enhanced photocatalytic ability.

Although the $\mathrm{Mg}$ ferrite particles, like their $\mathrm{Zn}$ ferrite analogues, evinced lower absorbance behavior in the UV-visible region, we found that they possessed the highest activity. We utilized Brunauer-Emmett-Teller (BET) analysis to determine the accessible 
surface area of the nanoparticles in order to correlate this variable with activity. BET analysis (Table 1) suggests that there is however no simple connection between surface area and photocatalytic activity. For example, highly active $\mathrm{Mg} 1$ with a surface area of $27.4 \mathrm{~m}^{2} / \mathrm{g}$ possesses a significantly lower surface area than many of the other ferrite nanoparticles. Our data moreover show the relative irrelevance of the choice of metal precursors themselves used to generate our metal ferrites, as the resulting photocatalytic properties are not directly correlated (Figure S14). Hence, our results suggest that for photocatalysis, the identity of the metal ' $M$ ' may actually in fact be more important than surface area and precursor considerations, presumably through the comparative, differential strengths of various ligandsubstrate interactions. Nevertheless, the implication is that one should be able to reliably tune for enhanced activities, primarily by controlling overall ferrite chemical composition. Moreover, we have also demonstrated that these ferrites can be potentially deployed as magnetically separable catalysts, as they are highly responsive to the application of an external magnetic field (Figure S15).

\section{(ii). Peroxidase-like activity}

Previously, Gao et al. showed that $\mathrm{Fe}_{3} \mathrm{O}_{4}$ nanoparticles possessed the ability to mimic the enzymatic activity similar to peroxidases, ${ }^{98}$ enzymes that can catalyze the oxidation of a variety of organic colorimetric substrates such as 3, 3', 5, 5' -tetramethylbenzidine (TMB). In many cases, the oxidation results in a color change (in our specific experiments, blue), which is typically utilized for immunoassays, sensing, and other detection methods, thereby highlighting the versatility of these materials for applications in biology, medicine, and environmental chemistry. The peroxidase enzymes function by forming a complex with and then subsequently decomposing hydrogen peroxide, which in turn oxidizes the substrate. Interestingly, many peroxidase enzymes rely on an iron-based heme group as the catalytic center. Hence, it is not surprising that iron oxide nanoparticles possess the ability to catalyze the decomposition of $\mathrm{H}_{2} \mathrm{O}_{2}$. In this cited work, the group investigated the catalytic roles of 
$\mathrm{Fe}^{2+}$ and $\mathrm{Fe}^{3+}$, which are both known to catalyze the decomposition of $\mathrm{H}_{2} \mathrm{O}_{2}$, and their findings suggested that the $\mathrm{Fe}^{2+}$ ions are more dominant in peroxidase-like catalysis as opposed to $\mathrm{Fe}^{3+}$ ions. By contrast, in our particular systems, the spinel ferrites lack the presence of $\mathrm{Fe}^{2+}$, and instead possess other $\mathrm{M}^{2+}$ ions. Previously, $\mathrm{CoFe}_{2} \mathrm{O}_{4}{ }^{99}$ and $\mathrm{ZnFe}_{2} \mathrm{O}_{4}{ }^{100}$ particles have been shown to display peroxidase-like activity. Hence, we have tried to investigate the potential of our ferrite nanoparticles as peroxidase mimics, and to the best of our knowledge, we are the first to report on the activity of ferrite nanoparticles as a function of chemical composition and particle size.

In effect, by comparison with our $\mathrm{Fe}_{3} \mathrm{O}_{4}$ nanoparticles, we found that many of our ferrite nanoparticles possess noticeably enhanced peroxidase-like activities, as shown in Figure 10, involving monitoring TMB absorbance variations at $652 \mathrm{~nm}$ as a function of catalytic reaction time. We find that our $\mathrm{MgFe}_{2} \mathrm{O}_{4}$ and $\mathrm{CuFe}_{2} \mathrm{O}_{4}$ samples as well as the 23.5 $\mathrm{nm}$ and $14.9 \mathrm{~nm} \mathrm{CoFe} \mathrm{O}_{4}$ nanoparticles displayed the highest activities. Moreover, ultra-small ferrite nanoparticles of $\mathrm{Ni}$ and $\mathrm{Co}$ also evinced enhanced activities by comparison with iron oxide (Figure 10 and S16, respectively). Interestingly, both sizes of Zn ferrite yielded very low peroxidase activity as compared with the $3.0 \mathrm{~nm}$ Ni ferrite sample, a finding which stands in contrast with the previous report by Su et al. ${ }^{100}$ Overall, these observations suggested that other $\mathrm{M}^{2+}$ ions may be more efficient at catalyzing the decomposition of $\mathrm{H}_{2} \mathrm{O}_{2}$ than the corresponding $\mathrm{Fe}^{2+}$ ions.

As with our data for photocatalysis, we attempted to correlate activity with the surface areas of our nanoparticles, as we postulated that it may also be playing a role, since many of the smaller sized nanoparticles displayed high activities. However, the ultra-small particles, although they possessed the largest surface areas (Table 1), did not necessarily achieve the highest peroxidase-like activity of all the samples tested. This observation again suggests that the ferrite chemical composition may be the more significant parameter in this reaction. Moreover, we postulate that although more surface sites were likely available, the small size 
of these ultra-small particles may have led to decreased activity due to either aggregation, poor accessibility, or surface passivation issues, thereby decreasing the overall reaction rate with the TMB substrate as opposed to the larger, more active ferrite nanoparticles.

Interestingly, we can correlate our Mössbauer spectroscopy results (Table 3), specifically the DOI values, to gain some additional insight into our observed catalytic enhancements. For example, samples $\mathrm{Co} 1$ and $\mathrm{Co} 2$ possess low degrees of inversion, indicating a predominance of $\mathrm{Co}^{2+}$ species at the nanoparticle surface. This inference comes from the fact that a majority of surface sites are (A)-type sites due to the lower metal ion coordination number at the surface, as discussed earlier. Thus, these two systems, demonstrating notable enhancements in peroxidase-like activity, suggest that $\mathrm{Co}^{2+}$ is a more active catalytic species as compared with $\mathrm{Fe}^{2+}$. Similarly, samples $\mathrm{Mg} 1$ and $\mathrm{Cu} 1$ were also noted to yield a majority of $\mathrm{M}^{2+}$ ions at their surfaces with correspondingly higher peroxidaselike activities as compared with iron itself. On the other hand, both as-prepared $\mathrm{ZnFe}_{2} \mathrm{O}_{4}$ samples, which also maintain lower degrees of inversion, yielded lower catalytic activities as compared with $\mathrm{Fe}_{3} \mathrm{O}_{4}$ itself, suggesting that $\mathrm{Zn}^{2+}$ in particular is a less active species. The same behavior was also observed for our nickel ferrite samples.

Hence, the key point of our experiments is that not only are ferrite nanoparticles active as peroxidase catalysts but also, depending on the chemical composition $\left(\mathrm{M}^{2+}\right)$ and the occupation of the surface sites of the specific ferrite system in question, many of our assynthesized nanoparticles are significantly more active than $\mathrm{Fe}_{3} \mathrm{O}_{4}$ alone at catalyzing the decomposition of $\mathrm{H}_{2} \mathrm{O}_{2}$, thereby resulting in an appreciable enhancement of the observed peroxidase-like behavior. Additionally, peroxidase activity data associated with our ultrasmall 'amorphous ferrites' are described and highlighted in Figure S16.

\section{Conclusions}


We have developed a simple, efficient hydrothermal method for the synthesis of large quantities of pure single-crystalline $\mathrm{MFe}_{2} \mathrm{O}_{4}$ (where 'M' $=\mathrm{Mg}, \mathrm{Fe}, \mathrm{Co}, \mathrm{Ni}, \mathrm{Cu}$, and $\mathrm{Zn}$ ) nanoparticles. We have explored a variety of experimental parameters in order to reliably tailor our synthesis in order to generate different compositions, sizes, and morphologies. Of the parameters investigated, the nature of the precursors as well as the inclusion of surfactant represented the most critical factors to consider in terms of 'selecting' for chemical composition and particle size. Specifically, by including APTES into the reaction solution, we could generate reasonably monodisperse, ultra-small nanoparticles possessing diameters less than $4.0 \mathrm{~nm}$ in size. None of the other reaction variables explored were found to give rise to any significant, controllable effect upon the resulting sample morphology or size.

By this method, we have generated different compositions and sizes of ferrite nanoparticles, which have been investigated for potential applications as (1) photocatalysts and (2) biological catalysts. In discerning practical and relevant structure-property correlations to explain these data, we utilized a combination of SQUID and Mössbauer techniques to systematically correlate nanoparticle size and composition with the observed magnetic behavior of our nanoparticles.

In so doing, we discovered that superparamagnetism is only present in the ultra-small nanoparticles $(\leq 4.0 \mathrm{~nm})$, with very magnetic low saturation values. The presence of spin canting, uncompensated surface spins and magnetic anisotropy was observed for a majority of our samples; blocking temperatures and spin-freezing temperatures associated with these systems were also discussed. Mössbauer spectroscopy supported the SQUID data and revealed that the occupancies of the tetrahedral $\mathrm{Fe}(\mathrm{A})$ and octahedral $\mathrm{Fe}[\mathrm{B}]$ sites were significantly altered, thereby emphasizing the importance of the synthetic method, size, and chemical composition.

Moreover, our data are consistent with prior observations reported by other groups ${ }^{44}$ 101, 102 who have noted that (a) differential ligand-surface interactions may be responsible for 
altering the observed surface spin-canting disorder and that (b) $T_{\mathrm{B}}$ as well as $M_{\mathrm{r}} / M_{\mathrm{s}}$ and $H_{\mathrm{c}}$ values generally increase with increasing nanoparticle size.

PDF results indicate that the smaller nanoparticle samples are significantly more disordered, but possess the same underlying spinel structure. It is not immediately clear why their magnetic behavior is so strongly modified, though we note that this is the same size where magnetic superexchange interactions associated with the spinel structure were observed to fail by Mössbauer spectroscopy at the particle's critical exchange length scale (vide supra).

We also investigated our metal ferrite nanoparticles for potential application as (1) photocatalysts and (2) peroxidase-like catalysts. Our exploration on the photodegradation of MB with our ferrite nanoparticles evinced that they are indeed photocatalytically active under UV irradiation. We found that of the ferrites tested, the $32.6 \mathrm{~nm} \mathrm{MgFe}_{2} \mathrm{O}_{4}$ nanoparticles possessed the highest activity. Moreover, similarly-sized nanoparticles gave rise to different

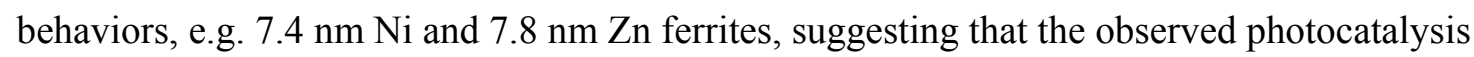
is primarily dependent on ' $\mathrm{M}$ '. By comparison with the available literature $(1.0 \mathrm{~g} / \mathrm{L})$, we have demonstrated that our as-prepared ferrite nanoparticles overall possess enhanced photocatalytic properties at much lower concentrations $(0.25 \mathrm{~g} / \mathrm{L})$.

Similarly, we also found that our ferrite nanoparticles are active as peroxidase-like catalysts. Specifically, samples of $\mathrm{MgFe}_{2} \mathrm{O}_{4}$ and $\mathrm{CuFe}_{2} \mathrm{O}_{4}$ nanoparticles as well as $23.5 \mathrm{~nm}$ and $14.9 \mathrm{~nm} \mathrm{CoFe} \mathrm{O}_{4}$ nanoparticles evinced very high activities towards TMB oxidation as compared with $\mathrm{Fe}_{3} \mathrm{O}_{4}$. Additionally, some of our ultra-small Ni and Co ferrite nanoparticles also displayed enhanced activities. To explain these data, by utilizing the degree of inversion obtained from our Mössbauer spectroscopy data, we were able to quantify the relative amounts of $\mathrm{M}^{2+}$ versus $\mathrm{Fe}^{2+}$ ions at the surface, thereby elucidating that both chemical composition and surface site occupancies play a significant role in catalytic activity.

Overall, these experiments highlight that our hydrothermal method generates robust, multifunctional, and fundamentally useful ferrite nanoparticles. The fact that we can tailor not 
only their size and degree of crystallinity, especially in the ultra-small range below $3 \mathrm{~nm}$, but also their chemical composition to a large degree therefore renders these ferrites as a highly versatile family of valuable materials, which are not only practically advantageous from an applications' point-of-view but also crystallographically significant from a fundamental science perspective.

\section{Acknowledgements}

Research (including support for ALT, CSL, JH, CZ, QL, SJLB, and SSW) was funded by the U.S. Department of Energy, Basic Energy Sciences, Materials Sciences and Engineering Division at Brookhaven National Laboratory, which is supported by the U.S. Department of Energy under Contract No. DE-AC02-98CH10886. We also thank James Quinn, Susan van Horn, and Vyacheslav (Steve) Volkov for their assistance with obtaining electron microscopy and energy dispersive X-ray analysis measurements. We also acknowledge Martin Schoonen for use of his BET equipment. Georgia Papaefthymiou thanks the U.S. National Science Foundation for support at Villanova University and the Ministry of Education of Brazil for financial support at the Brazilian Center for Research in Physics (Centro Brasileiro de Pesquisas Físicas, CBPF), in Rio de Janeiro, Brazil (CAPES visiting professorship of the exterior award) and Prof. Rosa Scorzelli for hosting her sabbatical leave at CBPF. The PDF data collection and analysis was supported by the Columbia University Energy Frontier Research Center (EFRC) funded by the U.S. Department of Energy Basic Energy Sciences program (DOE BES) under Grant No. DE-SC0001085. Use of the NSLS is supported by the U.S. Department of Energy, Basic Energy Sciences (DOE-BES) under contract number DE-AC02-98-CH10886. The ePDF data collection and analysis were performed in part at the Center for Functional Nanomaterials, located at Brookhaven National Laboratory, which is supported by the U.S. Department of Energy under contract number DEAC02-98CH10886. 


\section{Supporting Information Available:}

In addition to photocatalysis data, a table of samples systematically prepared by altering a number of reaction variables and the corresponding images for the synthesis optimization as a function of surfactant, reaction time, $\mathrm{NaOH}$ concentration, and reaction temperature as well as additional characterization data from PDF measurements in addition to results from low-temperature Mössbauer, magnetization, FT-IR, UV-visible, and EDS spectroscopies for representative ferrite nanoparticles. This material is available free of charge via the Internet at http://pubs.acs.org. 
Table 1. Reaction specifics, product characterization data, and surface area for $\mathrm{MFe}_{2} \mathrm{O}_{4}$ nanoparticles. All reactions were conducted at $190^{\circ} \mathrm{C}$ for 12 hours without the presence of surfactant, unless explicitly specified under 'Additional Notes'.

\begin{tabular}{|c|c|c|c|c|}
\hline 'M' & Sample \# & $\begin{array}{c}\text { Product } \\
\text { Characterization } \\
\text { (morphology and size) }\end{array}$ & Additional Notes & $\begin{array}{c}\text { Surface } \\
\text { area } \\
\left(\mathrm{m}^{2} / \mathrm{g}\right) \\
\end{array}$ \\
\hline $\mathrm{Mg}$ & 1 & $\begin{array}{c}\text { particles; } \\
32.9 \pm 17.9 \mathrm{~nm}\end{array}$ & $\mathrm{MgB}_{2}$ precursor & 27.4 \\
\hline \multirow{3}{*}{$\mathrm{Fe}$} & 1 & $\begin{array}{c}\text { cubes; } \\
68.9 \pm 42.7 \mathrm{~nm}\end{array}$ & $\mathrm{FeCl}_{2} \cdot 4 \mathrm{H}_{2} \mathrm{O}$ precursor & 9.87 \\
\hline & 2 & $\begin{array}{c}\text { particles; } \\
4.0 \pm 1.0 \mathrm{~nm}\end{array}$ & $\begin{array}{c}\mathrm{FeCl}_{2} \cdot 4 \mathrm{H}_{2} \mathrm{O} \text { precursor, } \\
1 \mathrm{~mL} \text { of APTES used; } \\
\text { amorphous }\end{array}$ & 183 \\
\hline & 3 & $\begin{array}{c}\mathrm{Fe}_{2} \mathrm{O}_{3} ; \text { spheres; } \\
222 \pm 38 \mathrm{~nm}\end{array}$ & $\mathrm{FeCl}_{3}$ precursor & - \\
\hline \multirow[b]{2}{*}{$\mathrm{Co}$} & 1 & $\begin{array}{c}\text { particles; } \\
23.5 \pm 4.9 \mathrm{~nm}\end{array}$ & $\mathrm{CoCl}_{2} \cdot 7 \mathrm{H}_{2} \mathrm{O}$ precursor & 78.7 \\
\hline & 2 & $\begin{array}{c}\text { particles; } \\
14.9 \pm 3.6 \mathrm{~nm}\end{array}$ & $\begin{array}{c}\mathrm{CoCl}_{2} \cdot 7 \mathrm{H}_{2} \mathrm{O} \text { precursor, } \\
1 \mathrm{~mL} \text { of NP- } 9^{[\mathrm{cc}]} \text { used }\end{array}$ & 54.3 \\
\hline \multirow{3}{*}{$\mathrm{Ni}$} & 1 & $\begin{array}{c}\text { cubes; } \\
87.3 \pm 26.2 \mathrm{~nm}\end{array}$ & $\mathrm{NiSO}_{4}$ precursor & 18.0 \\
\hline & 2 & $\begin{array}{c}\text { particles; } \\
7.4 \pm 2.5 \mathrm{~nm}\end{array}$ & $\mathrm{Ni}(\mathrm{acac})_{2}{ }^{[\mathrm{d}]}$ precursor & 59.4 \\
\hline & 3 & $\begin{array}{c}\text { particles; } \\
3.0 \pm 0.6 \mathrm{~nm}\end{array}$ & $\begin{array}{l}\mathrm{NiSO}_{4} \text { precursor, } 1 \mathrm{~mL} \\
\text { of APTES }{ }^{[\mathrm{b}]} \text { used; } \\
\text { run at } 220^{\circ} \mathrm{C} \\
\end{array}$ & 345.4 \\
\hline $\mathrm{Cu}$ & 1 & $\begin{array}{c}\text { particles; } \\
13.3 \pm 3.9 \mathrm{~nm}\end{array}$ & $\begin{array}{c}\mathrm{Cu}\left(\mathrm{NO}_{3}\right)_{2} \cdot 3 \mathrm{H}_{2} \mathrm{O} \\
\text { precursor }\end{array}$ & 5.8 \\
\hline \multirow[b]{2}{*}{$\mathrm{Zn}$} & 1 & $\begin{array}{c}\text { particles; } \\
7.8 \pm 2.0 \mathrm{~nm}\end{array}$ & $\mathrm{ZnF}_{2}$ precursor & 132 \\
\hline & 2 & $\begin{array}{c}\text { Particles } \\
3.1 \pm 0.7 \mathrm{~nm}\end{array}$ & $\begin{array}{l}\mathrm{ZnCl}_{2} \text { precursor, } 1 \mathrm{~mL} \\
\text { of } \mathrm{APTES}{ }^{[\mathrm{b}]} \text { used; } \\
\text { run at } 220^{\circ} \mathrm{C}\end{array}$ & 239.2 \\
\hline
\end{tabular}

[a] 'particles' refers to a spherical morphology, [b] APTES = 3-aminopropyltriethoxysilane, [c] NP9 = Igepal CO-630, and [d] (acac) = acetylacetonate. 

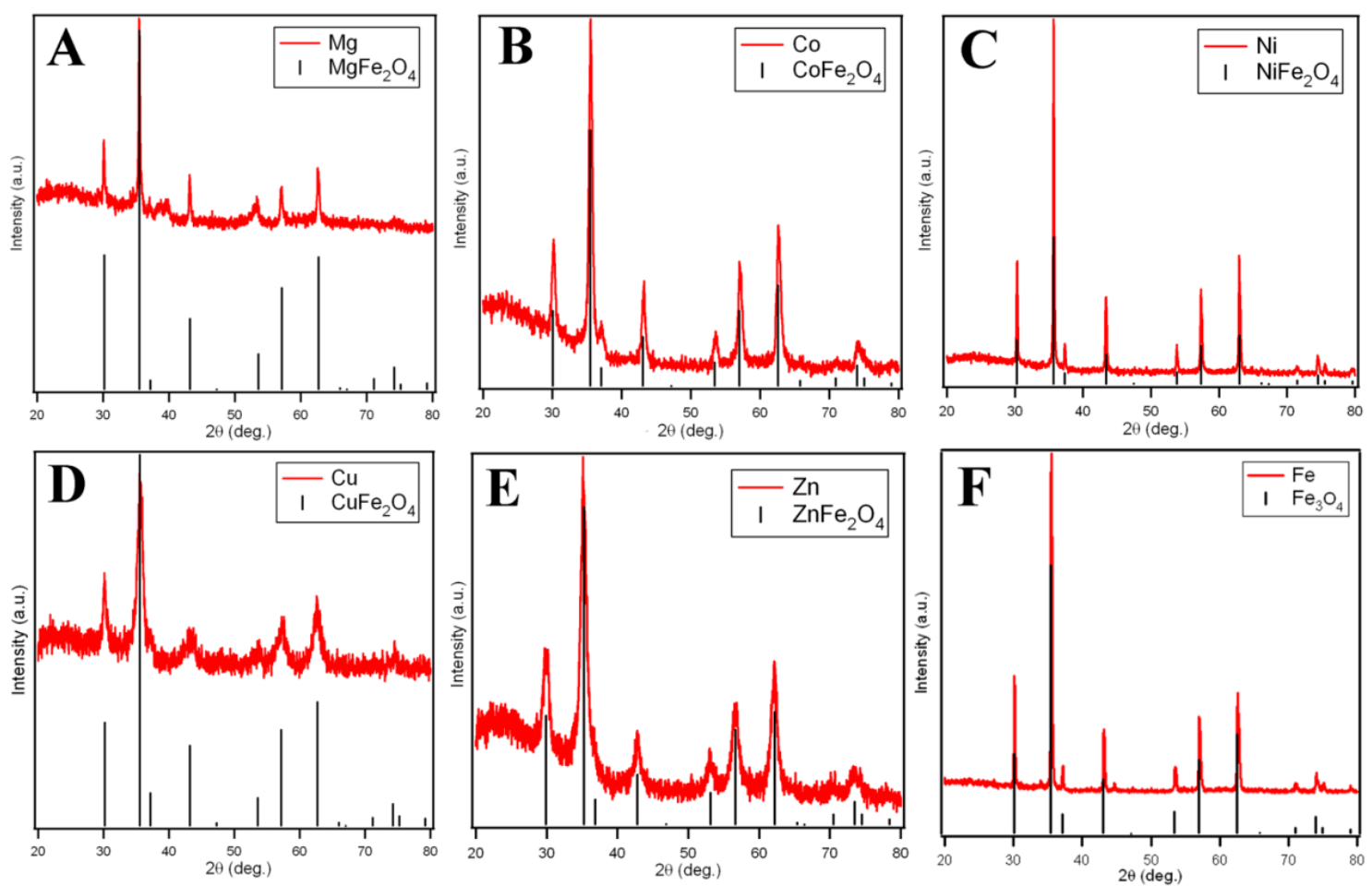

Figure 1. XRD data (red, upper curves) associated with the pure, crystalline $\mathrm{MFe}_{2} \mathrm{O}_{4}$ products synthesized by optimizing the metal precursors used, wherein ' $\mathrm{M}$ ' is $\mathrm{Mg}$ (A, Sample Mg1), Co (B, Sample Co1), Ni (C, Sample Ni1), Cu (D, Sample Cu1), Zn (E, Sample Zn1), and Fe (F, Sample Fe1). The JCPDS standards (black, lower curves) used to index the ferrites are \#732410, 22-1086, 74-2081, 77-0010, 82-1049, and 85-1436 for Mg, Co, Ni, Cu, Zn, and Fe ferrites, respectively. 


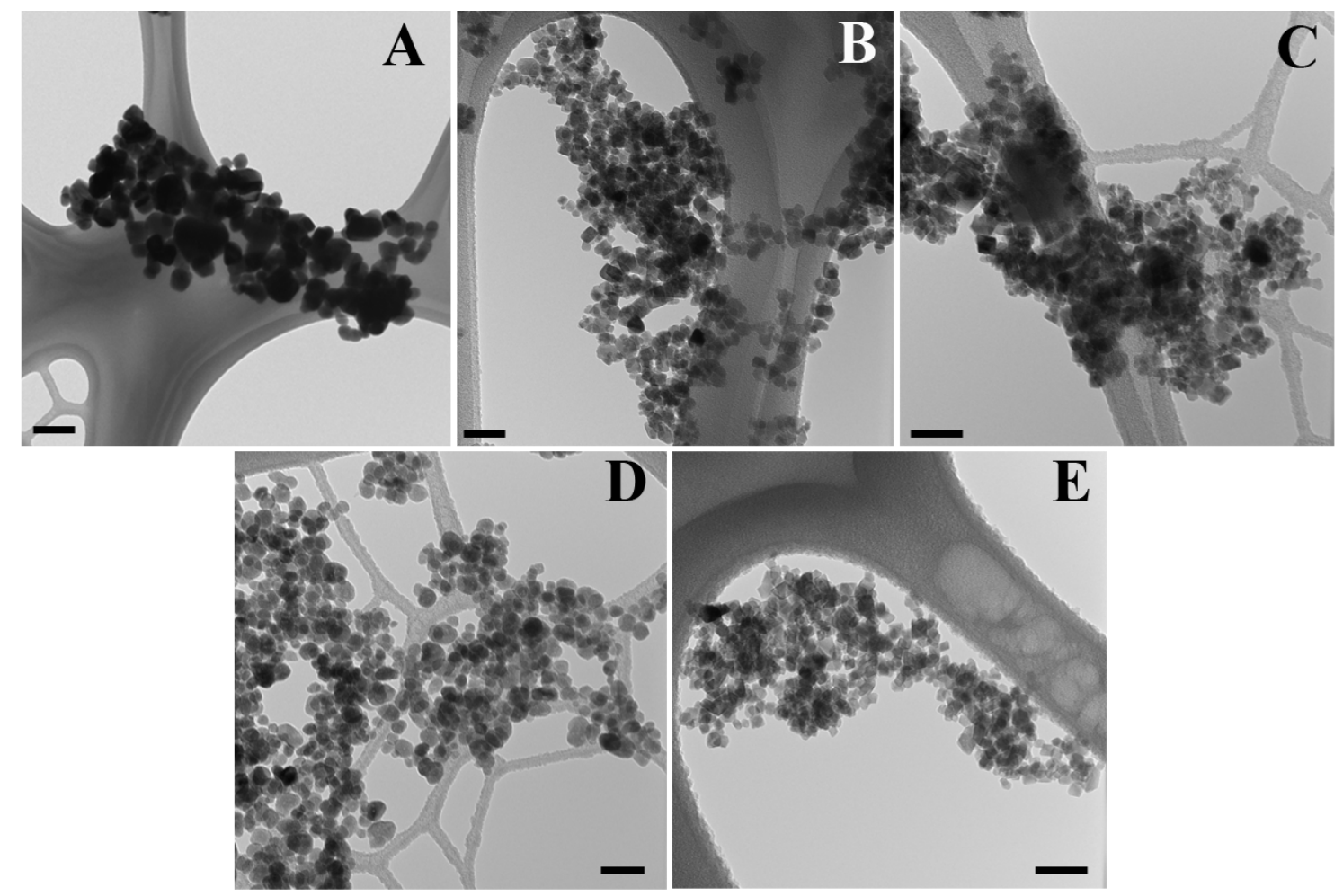

Figure 2. Representative TEM images associated with magnesium (A, sample $\mathrm{Mg} 1$ ), cobalt (B, sample Co2), nickel (C, sample Ni2), copper (D, sample Cu1), and zinc (E, sample Zn1) ferrite nanoparticles. All scale bars represent $40 \mathrm{~nm}$. 


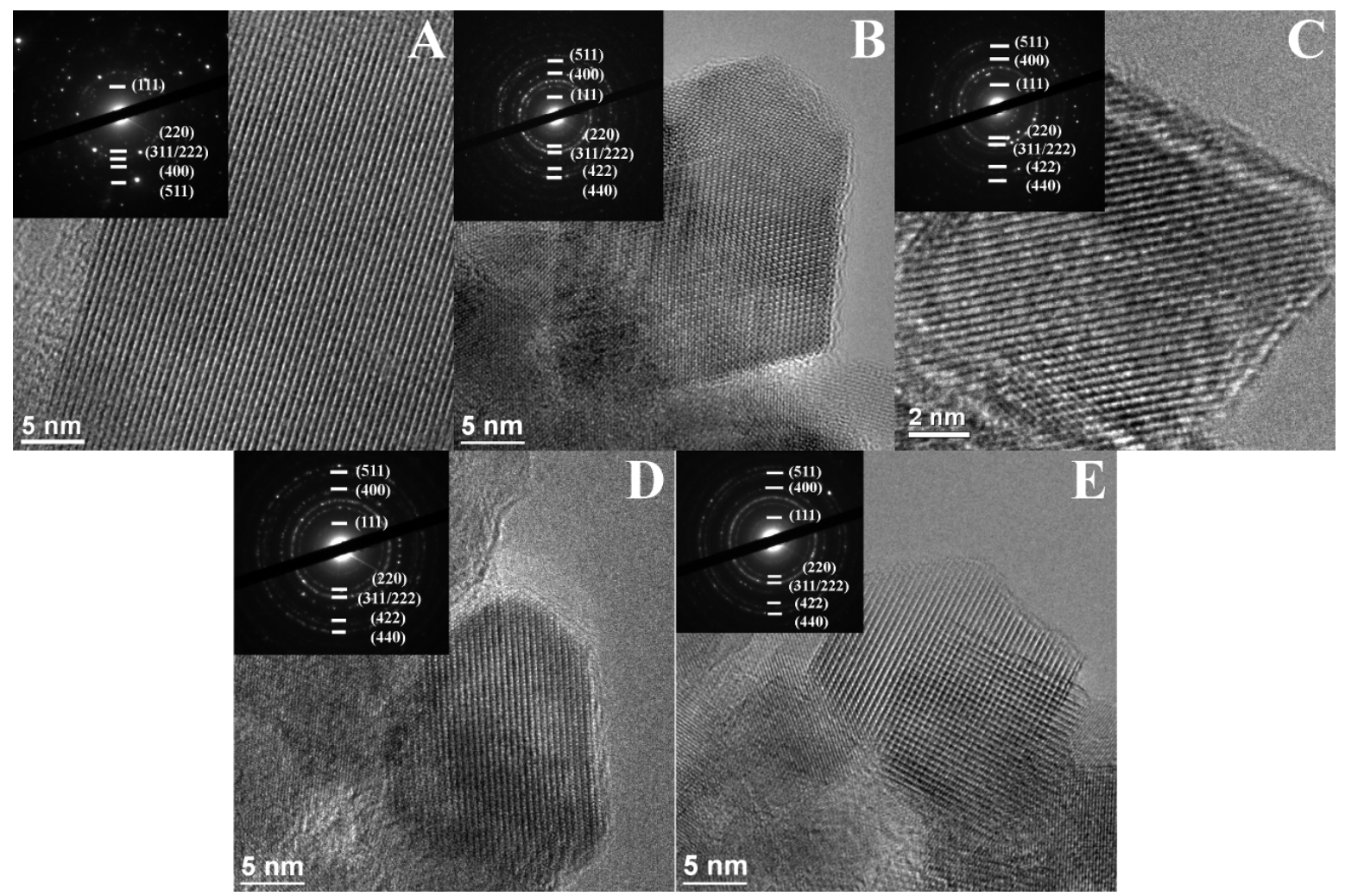

Figure 3. Representative high-resolution TEM images showing single-crystalline nanoparticles of magnesium (A, sample Mg1), cobalt (B, sample Co2), nickel (C, sample Ni2), copper (D, sample $\mathrm{Cu} 1)$, and zinc ferrite nanoparticles (E, sample Zn1). The selected-area electron diffraction patterns (A-E insets) can be indexed to the cubic spinel structure of the ferrites. All scale bars are $5 \mathrm{~nm}$, except for nickel ferrite (C) in which the scale bar represents $2 \mathrm{~nm}$. 

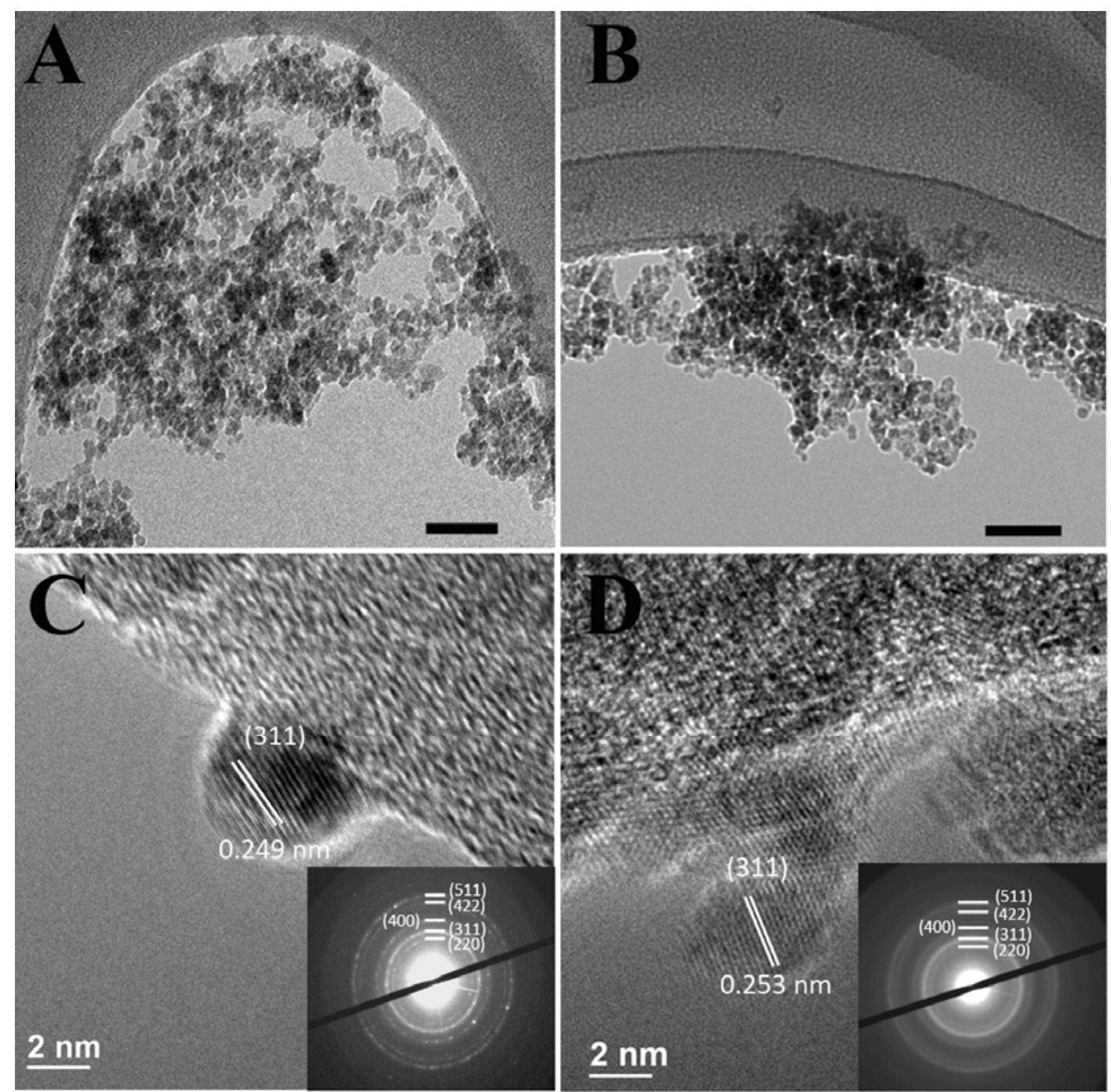

Figure 4. TEM images associated with nickel (A, Ni3) and zinc (B, Zn2) metal ferrites prepared at $220^{\circ} \mathrm{C}$. High-resolution TEM images showing single-crystalline nanostructures of nickel (C, sample Ni3) and zinc (D, sample Zn2) metal ferrite samples. All scale bars associated with low resolution TEM are $40 \mathrm{~nm}$. The selected-area electron diffraction patterns (C-D insets) can be indexed to the cubic spinel structure of the ferrites. 

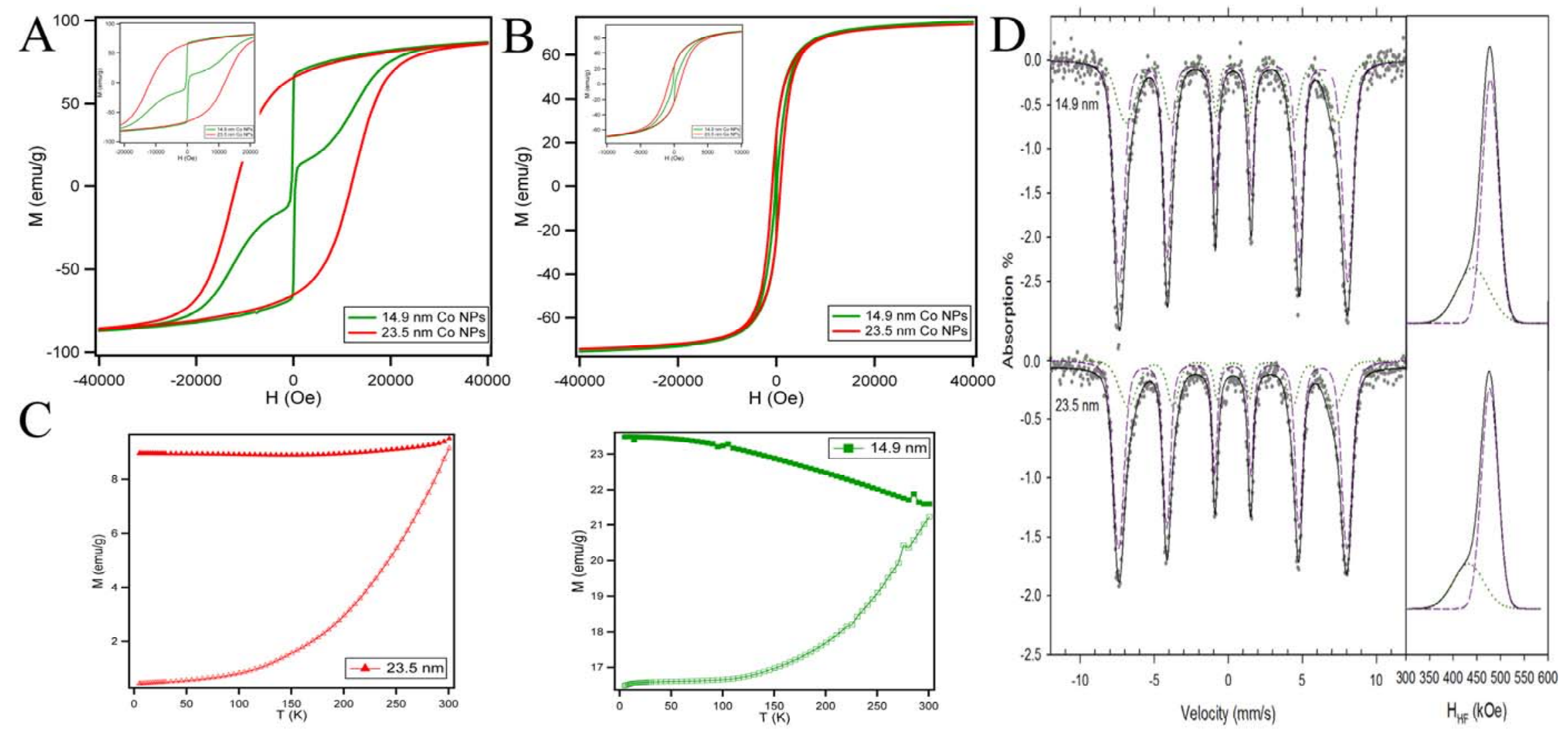

Figure 5. $\mathrm{M}$ versus $\mathrm{H}$ curves at $5 \mathrm{~K}(\mathrm{~A})$ and $300 \mathrm{~K}(\mathrm{~B})$ with insets showing a magnified view along with ZFC-FC curves at an applied field of 500 $\mathrm{Oe}(\mathrm{C})$ for the $23.5 \mathrm{~nm}$ (red) and $14.9 \mathrm{~nm}$ (green) $\mathrm{CoFe}_{2} \mathrm{O}_{4}$ nanoparticles, along with room temperature Mössbauer spectra (D) with the solid black line, dotted green line, and dashed purple line corresponding to the theoretical fit, contribution of the $\mathrm{Fe}(\mathrm{A})$, and contribution of the Fe[B] sites, respectively. (D). Left panel: Mössbauer spectra of Co1 (bottom) and Co2 (top) at RT fitted to a superposition of two groups of magnetic hyperfine fields associated with (A) (green) and [B] (purple) sites. Right panel: Derived hyperfine field distributions for each subsite, (A) green line, [B] purple line, overall, black line, assuming Gaussian distributions. 
Table 2. Magnetic characterization data associated with $\mathrm{MFe}_{2} \mathrm{O}_{4}$ nanoparticles, obtained from $\mathrm{M}$ versus $\mathrm{H}$ curves at $5 \mathrm{~K}$ and $300 \mathrm{~K}$, along with ZFC-FC curves at an applied field of $500 \mathrm{Oe}$. Note that the unit emu/g is calculated for the total mass of the material.

\begin{tabular}{|c|c|c|c|c|c|c|c|}
\hline Sample & Size (nm) & $\mathbf{T}(\mathrm{K})$ & $\begin{array}{c}H_{\mathrm{C}} \\
(\mathbf{O e})\end{array}$ & $\begin{array}{c}M_{\mathrm{S}} \\
(\mathrm{emu} / \mathrm{g})\end{array}$ & $\begin{array}{c}M_{\mathrm{R}} \\
(\mathrm{emu} / \mathrm{g})\end{array}$ & $M_{\mathrm{R}} / M_{\mathrm{S}}$ & $\underset{(\mathrm{K})}{T_{\max } / T_{\mathrm{B}}}$ \\
\hline \multirow{2}{*}{ Mg1 } & \multirow{2}{*}{32.6} & 5 & 339 & 43.8 & 9.56 & 0.22 & \multirow{2}{*}{$>300$} \\
\hline & & 300 & 77 & 34.0 & 3.86 & 0.11 & \\
\hline \multirow{2}{*}{ Col } & \multirow{2}{*}{23.5} & 5 & 11842 & 85.3 & 65.52 & 0.77 & \multirow{2}{*}{$>300$} \\
\hline & & 300 & 849 & 74.5 & 23.44 & 0.31 & \\
\hline \multirow{2}{*}{$\mathrm{Co} 2$} & \multirow{2}{*}{14.9} & 5 & 444 & 88.4 & 54.12 & 0.61 & \multirow{2}{*}{$>300$} \\
\hline & & 300 & 179 & 75.9 & 21.68 & 0.29 & \\
\hline \multirow{2}{*}{ Ni1 } & \multirow{2}{*}{87.3} & 5 & 436 & 52.5 & 19.11 & 0.36 & \multirow{2}{*}{$>300$} \\
\hline & & 300 & 43 & 46.0 & 3.36 & 0.07 & \\
\hline \multirow{2}{*}{$\mathrm{Ni} 2$} & \multirow{2}{*}{7.4} & 5 & 364 & 52.0 & 16.8 & 0.32 & \multirow{2}{*}{270} \\
\hline & & 300 & 39 & 44.8 & 3.69 & 0.08 & \\
\hline \multirow{2}{*}{$\mathrm{Ni3}$} & \multirow{2}{*}{3.0} & 5 & 160 & 40.8 & 8.69 & 0.21 & \multirow{2}{*}{29} \\
\hline & & 300 & 17 & 12.1 & 0.19 & 0.01 & \\
\hline \multirow{2}{*}{$\mathrm{Cu} 1$} & \multirow{2}{*}{13.3} & 5 & 485 & 5.5 & 1.91 & 0.35 & \multirow{2}{*}{165} \\
\hline & & 300 & 2 & 4.5 & 0.02 & 0.004 & \\
\hline \multirow{2}{*}{$\mathrm{Zn} 1$} & \multirow{2}{*}{7.8} & 5 & 259 & 54.5 & 7.81 & 0.14 & \multirow{2}{*}{22} \\
\hline & & 300 & - & 15.2 & - & - & \\
\hline \multirow{2}{*}{$\mathrm{Zn} 2$} & \multirow{2}{*}{3.1} & 5 & 18.3 & 7.5 & 0.59 & 0.08 & \multirow{2}{*}{6} \\
\hline & & 300 & - & 6.4 & - & - & \\
\hline
\end{tabular}



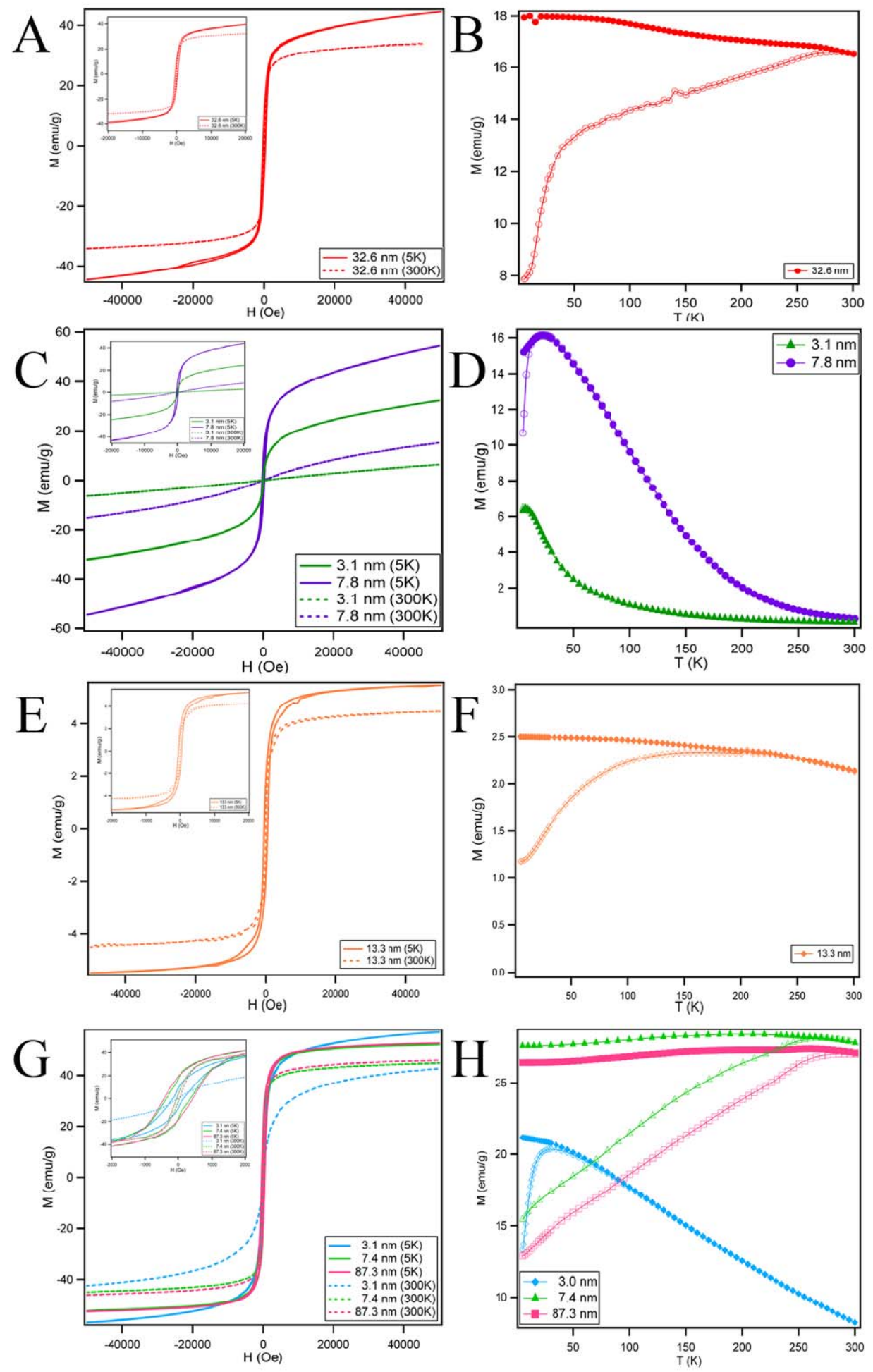

Figure 6. $\mathrm{M}$ versus $\mathrm{H}$ curves at $5 \mathrm{~K}$ and $300 \mathrm{~K}$ with insets showing a magnified view along with ZFC-FC curves at an applied field of 500 Oe for $32.6 \mathrm{~nm} \mathrm{MgFe}{ }_{2} \mathrm{O}_{4}$ (A, B), $3.0 \mathrm{~nm}$ and $7.8 \mathrm{~nm} \mathrm{ZnFe}{ }_{2} \mathrm{O}_{4}(\mathrm{C}, \mathrm{D})$, and $13.3 \mathrm{~nm} \mathrm{CuFe}_{2} \mathrm{O}_{4}$ (E, F) nanoparticles, as well as $3.1 \mathrm{~nm}, 7.4 \mathrm{~nm}$, and $87.3 \mathrm{~nm} \mathrm{NiFe} \mathrm{O}_{4}(\mathrm{G}, \mathrm{H})$ nanoparticles, respectively. FC and ZFC curves are shown as solid and open markers, respectively. Note that the unit emu/g is calculated for the total mass of the material. 
Table 3. Fitted ${ }^{57} \mathrm{Fe}$ Mössbauer parameters of selected $\mathrm{MFe}_{2} \mathrm{O}_{4}$ nanoparticles at $300 \mathrm{~K}$.

\begin{tabular}{|c|c|c|c|c|c|c|c|c|c|}
\hline Sample & $\begin{array}{l}\text { Size } \\
(\mathrm{nm})\end{array}$ & Site $^{[\mathrm{a}]}$ & $\begin{array}{c}\Gamma^{[\mathbf{b}]} \\
(\mathbf{m m} / \mathbf{s})\end{array}$ & $\begin{array}{c}\delta^{[\mathrm{c}]} \\
(\mathbf{m m} / \mathbf{s})\end{array}$ & $\begin{array}{c}\Delta \mathbf{E}_{\mathbf{Q}}^{[\mathrm{d}]} \\
(\mathrm{mm} / \mathbf{s})\end{array}$ & $\begin{array}{c}\mathbf{B}_{\mathrm{HF}}{ }^{[\mathrm{e}]} \\
(\mathrm{T})\end{array}$ & $\begin{array}{c}\text { HFD }^{[\mathbf{f}]} \\
(\mathbf{F W H M}) \\
(\mathrm{T})\end{array}$ & $\begin{array}{c}\operatorname{Area}^{[g]} \\
(\%)\end{array}$ & $\begin{array}{l}\mathrm{Fe}(\mathrm{A}) / \mathrm{Fe}[\mathrm{B}] \\
\text { (inversion) }^{[\mathrm{h}]}\end{array}$ \\
\hline \multirow{2}{*}{ Col } & \multirow{2}{*}{23.5} & 1 & 0.36 & 0.27 & - & 43.2 & 7.1 & 27 & \multirow{2}{*}{$0.37 \pm 0.09$} \\
\hline & & 2 & 0.36 & 0.30 & - & 47.9 & 4.1 & 73 & \\
\hline \multirow{2}{*}{$\mathrm{Co} 2$} & \multirow{2}{*}{14.9} & 1 & 0.36 & 0.25 & - & 44.1 & 8.1 & 31 & \multirow{2}{*}{$0.45 \pm 0.10$} \\
\hline & & 2 & 0.36 & 0.35 & - & 47.7 & 4.0 & 69 & \\
\hline \multirow{2}{*}{$\mathrm{Zn} 1$} & \multirow{2}{*}{7.8} & 1 & 0.34 & 0.35 & 0.61 & & - & 46 & \multirow{2}{*}{$0.85 \pm 0.17$} \\
\hline & & 2 & 0.27 & 0.35 & 0.35 & - & & 54 & \\
\hline \multirow{2}{*}{$\mathrm{Zn} 2$} & \multirow{2}{*}{3.1} & 1 & 0.35 & 0.35 & 0.82 & - & - & 37 & \multirow{2}{*}{$0.59 \pm 0.13$} \\
\hline & & 2 & 0.30 & 0.34 & 0.44 & - & - & 63 & \\
\hline \multirow{2}{*}{ Nil } & \multirow{2}{*}{87.3} & 1 & 0.31 & 0.26 & - & 48.4 & 2.8 & 56 & \multirow{2}{*}{$1.27 \pm 0.25$} \\
\hline & & 2 & 0.31 & 0.37 & - & 51.7 & 2.4 & 44 & \\
\hline \multirow{5}{*}{$\mathrm{Ni} 2$} & \multirow{5}{*}{7.4} & 1 & 0.66 & 0.28 & - & 49.9 & 2.1 & 46 & \multirow{5}{*}{$1.35 \pm 0.29$} \\
\hline & & 2 & 0.66 & 0.38 & - & 51.8 & 1.9 & 31 & \\
\hline & & SPD & 0.75 & 0.36 & 0.70 & - & - & 6 & \\
\hline & & 1(IR) & 0.50 & 0.31 & - & 27.0 & 15.0 & 8 & \\
\hline & & $2(\mathrm{IR})$ & 0.75 & 0.31 & - & 44.0 & 8.0 & 9 & \\
\hline \multirow{4}{*}{ Ni3 } & \multirow{4}{*}{3.0} & 1 & 0.50 & 0.30 & 0.86 & - & - & 20 & \multirow{4}{*}{$0.74 \pm 0.32$} \\
\hline & & 2 & 0.50 & 0.31 & 0.43 & - & - & 27 & \\
\hline & & 1(IR) & 0.36 & 0.33 & - & 2.0 & 15.2 & 47 & \\
\hline & & 2(IR) & 0.36 & 0.30 & - & 23.7 & 12.1 & 6 & \\
\hline \multirow{2}{*}{$\mathrm{Cu} 1$} & \multirow{2}{*}{13.3} & 1 & 0.55 & 0.33 & - & 45.8 & 7.8 & 22 & \multirow{2}{*}{$0.28 \pm 0.08$} \\
\hline & & 2 & 0.28 & 0.38 & - & 51.5 & 2.0 & 78 & \\
\hline \multirow{3}{*}{ Mg1 } & \multirow{3}{*}{32.6} & SPD & 0.55 & 0.39 & 0.80 & - & - & 36 & \multirow{3}{*}{1.06} \\
\hline & & 1 & 0.55 & 0.65 & - & 48.6 & 7.2 & 33 & \\
\hline & & 2 & 0.40 & 0.31 & - & 51.9 & 2.1 & 31 & \\
\hline
\end{tabular}

[a] Site $1=$ tetrahedral $\mathrm{Fe}(\mathrm{A})$ site, Site $2=$ octahedral Fe[B] site, SPD = superparamagnetic doublet, IR = intermediate relaxation [b] full width of absorption lines at half maximum with error $\pm 0.03 \mathrm{~mm} / \mathrm{s}$, [c] isomer shift relative to $\mathrm{Fe}$ at $300 \mathrm{~K}$ with error $\pm 0.03 \mathrm{~mm} / \mathrm{s}$, [d] quadrupole splitting with error \pm 0.05 , [e] hyperfine field with error of \pm 0.5 , [f] Hyperfine field distribution (full width half maximum), [g] area below the spectrum with error \pm 5 , and [h] degree of inversion with associated errors.. 


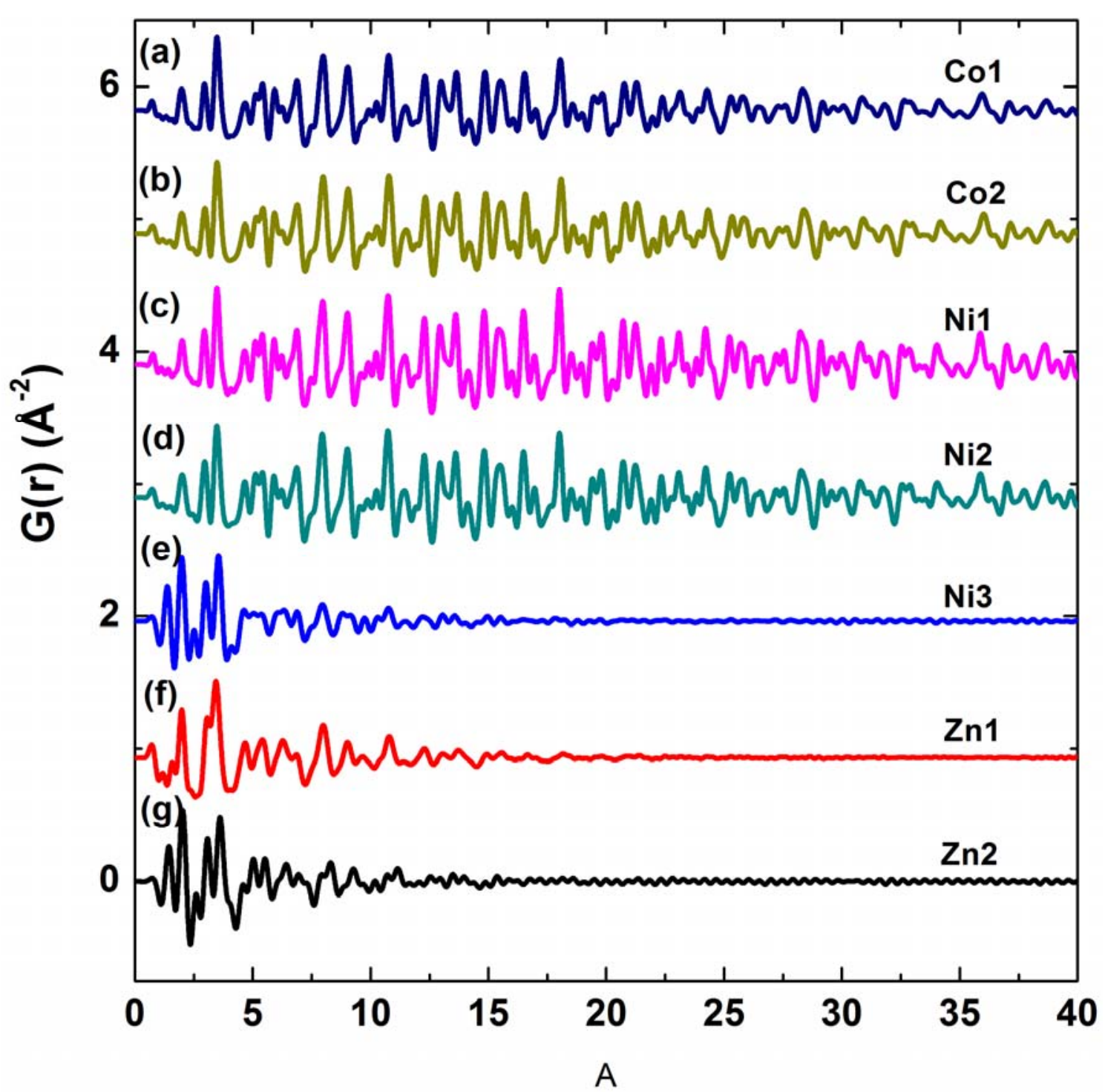

Figure 7. Measured PDFs for $\mathrm{Co}, \mathrm{Ni}$, and $\mathrm{Zn}$ ferrite nanoparticles of various sizes plotted in a $r$-range up to $40 \AA$. 


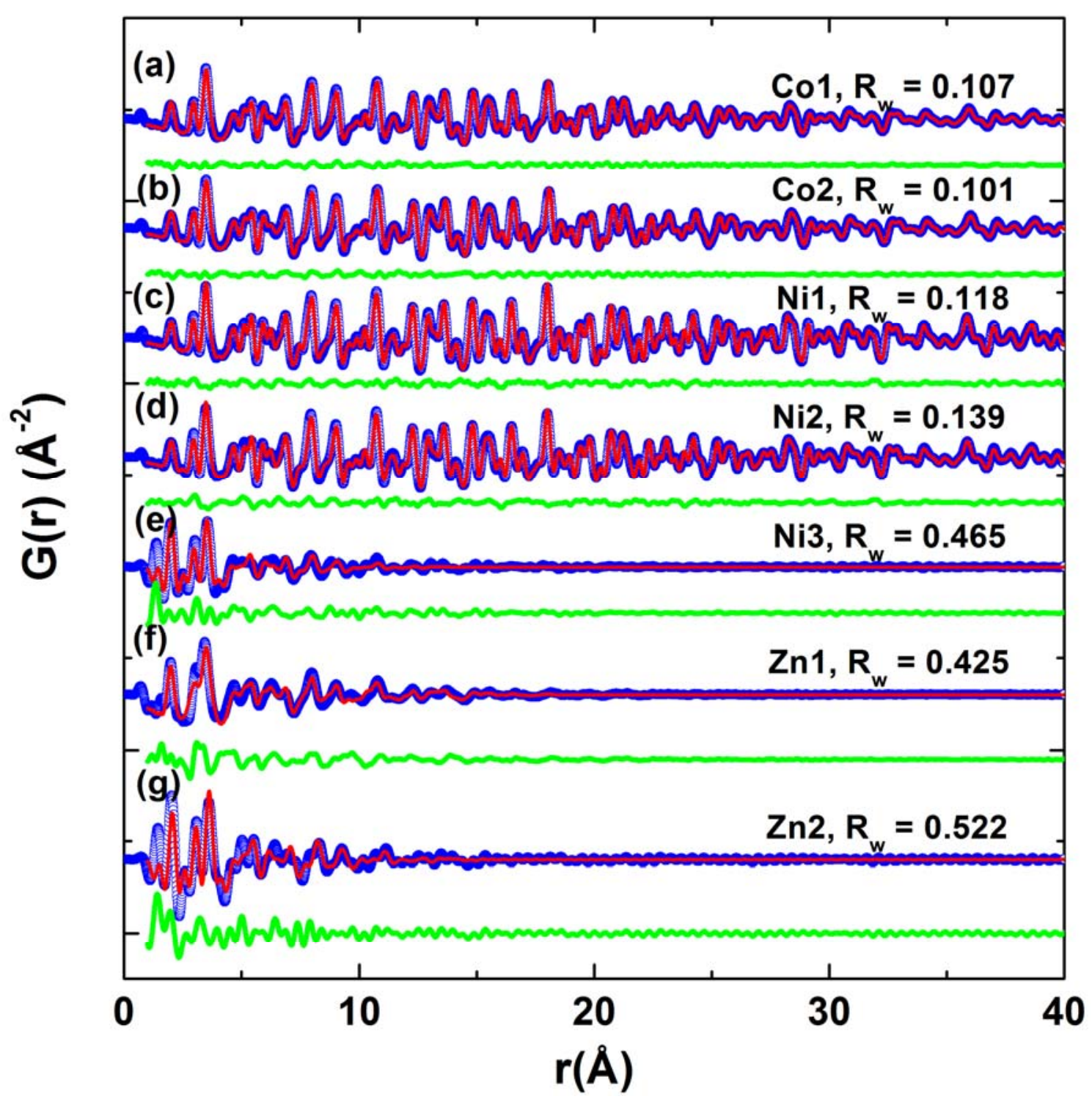

Figure 8. PDF fits for as-prepared $\mathrm{Co}, \mathrm{Ni}$, and $\mathrm{Zn}$ ferrite NPs in an 'r-range' from $1 \AA$ to 40 $\AA$. The blue circles and red solid lines correspond to measured and simulated PDFs, respectively. The green solid lines offset below are the difference curves. Agreement factors, $R_{w}$, are displayed beside each model fit. 


\begin{tabular}{|c|c|c|c|c|c|c|c|c|c|c|}
\hline - & $\mathbf{R}_{\mathbf{w}}$ & DOI $^{\mathrm{a}}$ & DOI $^{\mathrm{b}}$ & $a(\AA)$ & $z(\mathbf{O})$ & $U_{i s o}(M)\left(\AA^{2}\right)^{c}$ & $\mathrm{U}_{\text {iso }}(\mathrm{Fe})\left(\AA^{2}\right)$ & $\mathbf{U}_{\text {iso }}(\mathbf{O})\left(\AA^{2}\right)$ & $\begin{array}{c}\text { Structural } \\
\text { Coherence }(\AA)\end{array}$ & $\operatorname{Size}(\AA)^{\mathrm{e}}$ \\
\hline Co1 & 0.107 & --- & 0.540 & 8.365 & 0.256 & 0.0057 & 0.0077 & 0.0194 & $106^{\mathrm{f}}$ & $235(49)$ \\
\hline $\mathrm{Co} 2$ & 0.101 & --- & 0.621 & 8.381 & 0.256 & 0.0062 & 0.0067 & 0.0178 & $130.5^{\mathrm{f}}$ & $149(36)$ \\
\hline Ni1 & 0.118 & --- & 1.119 & 8.346 & 0.255 & 0.0037 & 0.0062 & 0.0120 & $1198.5^{\mathrm{f}}$ & $873(262)$ \\
\hline $\mathrm{Ni} 2$ & 0.139 & --- & 1.149 & 8.348 & 0.254 & 0.0030 & 0.0072 & 0.0120 & $174^{\mathrm{f}}$ & $74(25)$ \\
\hline Ni3 & 0.465 & --- & 0.561 & 8.417 & 0.262 & 0.0038 & 0.0066 & 0.0051 & 15.92 & $30(6)$ \\
\hline Zn1 & 0.425 & 0.509 & 0.850 & 8.367 & 0.256 & 0.0044 & 0.0762 & 0.0181 & 20.23 & $78(20)$ \\
\hline $\mathrm{Zn} 2$ & 0.522 & 1.209 & 0.590 & 8.660 & 0.265 & 0.0043 & 0.0048 & 0.0023 & 18.97 & $31(6)$ \\
\hline
\end{tabular}

Table 4. Summary of PDF fits associated with $\mathrm{Co}$, Ni, and $\mathrm{Zn}$ ferrite nanoparticles. For fits with $\mathrm{R}_{\mathrm{w}}$ values greater than $50 \%$ (Co3 and $\mathrm{Ni3}$ ), the fit results are not valid therefore not shown in the table.

${ }^{a}$ Degree of inversion values originate from PDF modeling.

${ }^{\mathrm{b}}$ Degree of inversion values come from the Mössbauer data (Table 3).

$c$ "M" stands for metal ions other than Fe. If the sample is $\mathrm{Fe}_{3} \mathrm{O}_{4}, \mathrm{M}$ is $\mathrm{Fe}$.

${ }^{\mathrm{d}}$ Structural coherence arises from PDF fit using a spherical characteristic function.

${ }^{\mathrm{e}}$ Particle sizes are obtained from transmission electron microscopy (TEM) measurements (Table 1).

${ }^{\mathrm{f}}$ The precision of these values is low and limited by the poor instrumental resolution. The range of structural coherence refined from PDF becomes less accurate the larger the particles are above $\sim 65 \AA$, which represents the radial distance where the signal of the bulk nickel calibration standard vanishes. 

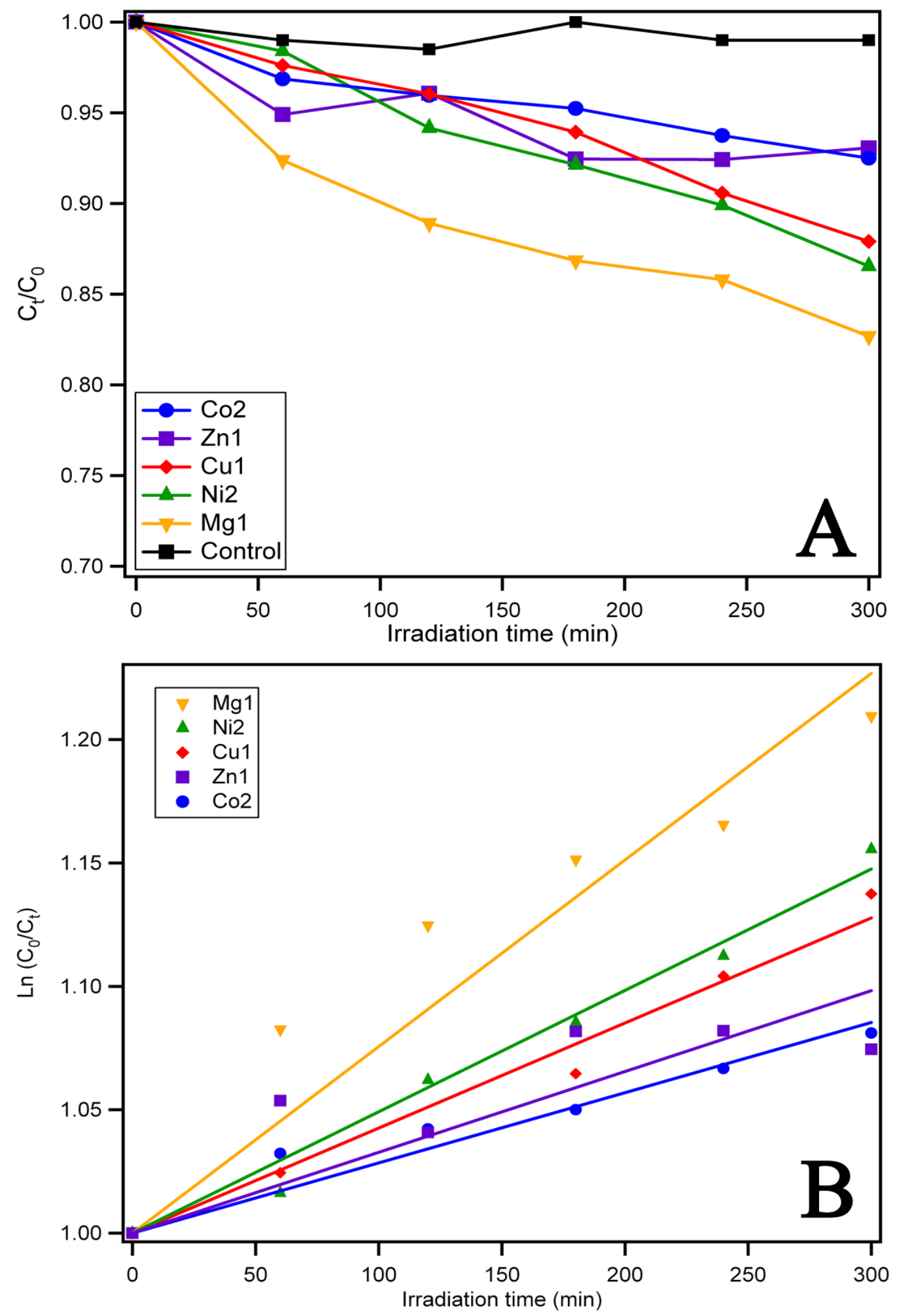

Figure 9. Photodegradation of methylene blue under $366 \mathrm{~nm} \mathrm{UV}$ irradiation in the presence of magnesium (yellow, $\boldsymbol{\nabla}$ ), nickel (green, $\mathbf{\Delta}$ ), copper (red, $\bullet$ ), zinc (purple, $\boldsymbol{\square}$ ), and cobalt $($ blue, $\bullet$ ) ferrite nanoparticles as a function of irradiation time as compared with a control (black, - ) of methylene blue without any nanoparticles present (A). Pseudo-first-order fits of the data as a logarithmic change in concentration as a function of UV irradiation time (B). 


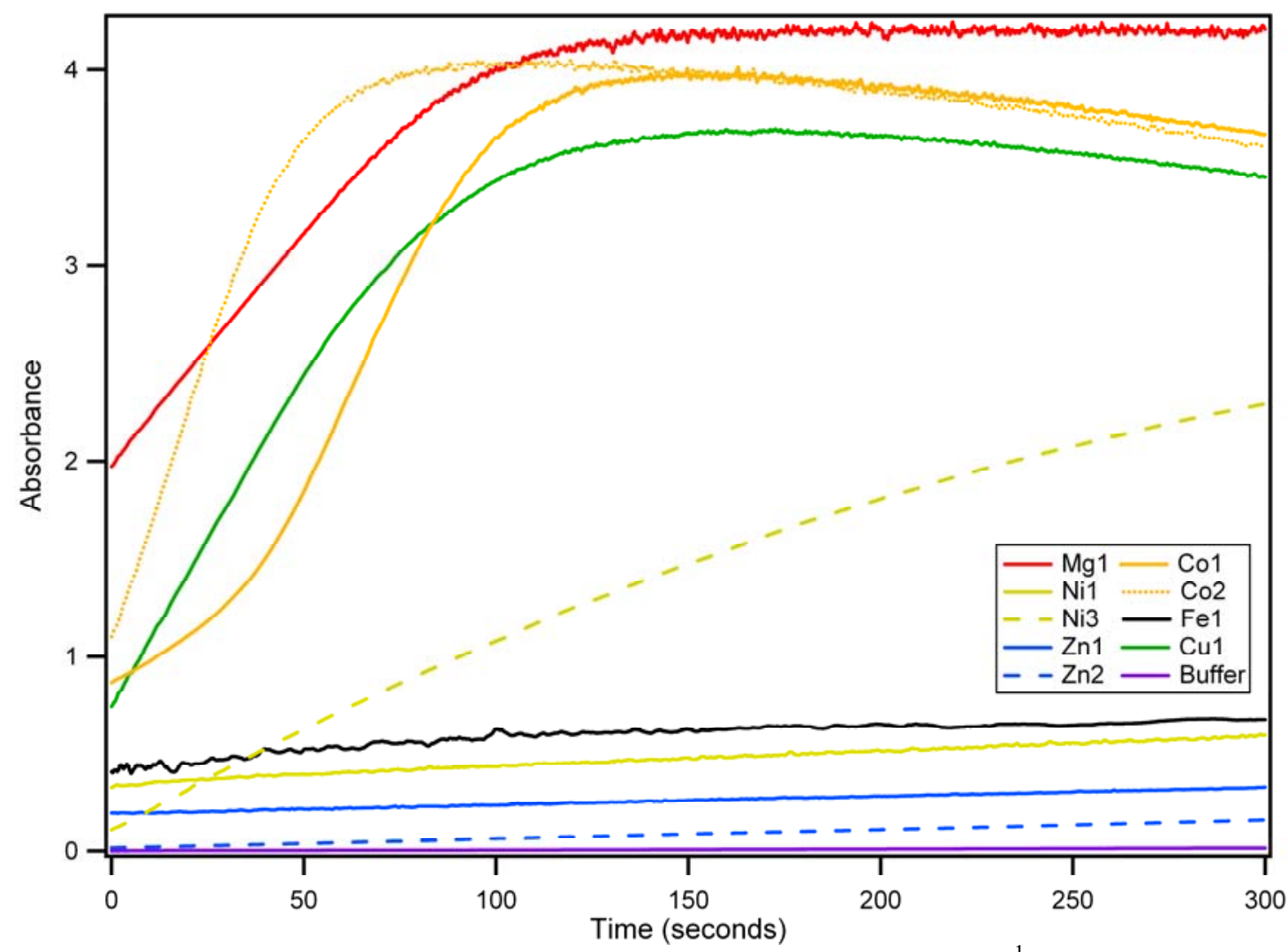

Figure 10. Peroxidase-like activity of ferrite nanoparticles (at $1 \mathrm{mg} \mathrm{mL}^{-1}$ concentration) towards TMB, measured at $652 \mathrm{~nm}$ as a function of reaction time. 


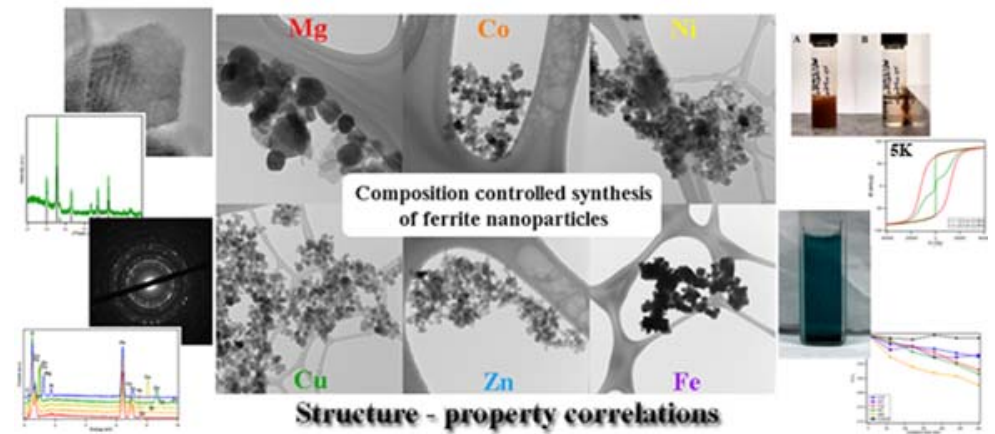

TOC Figure Caption. By generating ferrite nanoparticles of controlled sizes and compositions, variations in nanoscale structure can be rigorously correlated with magnetic and catalytic properties. 


\section{References}

1. Mathew, D. S.; Juang, R.-S. An overview of the structure and magnetism of spinel ferrite nanoparticles and their synthesis in microemulsions. Chemical Engineering Journal 2007, 129 (1-3), 51-65.

2. $\quad$ Sun, S.; Zeng, H.; Robinson, D. B.; Raoux, S.; Rice, P. M.; Wang, S. X.; Li, G. Monodisperse $\mathrm{MFe}_{2} \mathrm{O}_{4}(\mathrm{M}=\mathrm{Fe}, \mathrm{Co}, \mathrm{Mn})$ Nanoparticles. Journal of the American Chemical Society 2003, 126 (1), 273-279.

3. Kodama, R. H.; Berkowitz, A. E.; McNiff Jr., E. J.; Foner, S. Surface spin disorder in ferrite nanoparticles (invited). Journal of Applied Physics 1997, 81 (8), 5552-5557.

4. Pereira, C.; Pereira, A. M.; Fernandes, C.; Rocha, M.; Mendes, R.; Fernández-García, M. P.; Guedes, A.; Tavares, P. B.; Grenèche, J.-M.; Araújo, J. P.; Freire, C.

Superparamagnetic $\mathrm{MFe}_{2} \mathrm{O}_{4}(\mathrm{M}=\mathrm{Fe}, \mathrm{Co}, \mathrm{Mn})$ Nanoparticles: Tuning the Particle Size and

Magnetic Properties through a Novel One-Step Coprecipitation Route. Chemistry of Materials 2012, 24 (8), 1496-1504.

5. $\quad$ Lee, J.-H.; Jang, J.-t.; Choi, J.-s.; Moon, S. H.; Noh, S.-h.; Kim, J.-w.; Kim, J.-G.;

Kim, I.-S.; Park, K. I.; Cheon, J. Exchange-coupled magnetic nanoparticles for efficient heat induction. Nat Nano 2011, 6 (7), 418-422.

6. Jang, J.-t.; Nah, H.; Lee, J.-H.; Moon, S. H.; Kim, M. G.; Cheon, J. Critical Enhancements of MRI Contrast and Hyperthermic Effects by Dopant-Controlled Magnetic Nanoparticles. Angewandte Chemie 2009, 121 (7), 1260-1264.

7. Kim, D.-H.; Nikles, D. E.; Johnson, D. T.; Brazel, C. S. Heat generation of aqueously dispersed $\mathrm{CoFe}_{2} \mathrm{O}_{4}$ nanoparticles as heating agents for magnetically activated drug delivery and hyperthermia. Journal of Magnetism and Magnetic Materials 2008, 320 (19), 2390-2396.

8. Gadkari, A. B.; Shinde, T. J.; Vasambekar, P. N. Ferrite Gas Sensors. Sensors Journal, IEEE 2011, 11 (4), 849-861.

9. Zhang, G.; Li, C.; Cheng, F.; Chen, J. $\mathrm{ZnFe}_{2} \mathrm{O}_{4}$ tubes: Synthesis and application to gas sensors with high sensitivity and low-energy consumption. Sensors and Actuators B:

Chemical 2007, 120 (2), 403-410.

10. Ying, W.; Dawei, S.; Alison, U.; Jung-ho, A.; Guoxiu, W. Hollow $\mathrm{CoFe}_{2} \mathrm{O}_{4}$ nanospheres as a high capacity anode material for lithium ion batteries. Nanotechnology 2012, $23(5), 055402$.

11. Frey, N. A.; Peng, S.; Cheng, K.; Sun, S. Magnetic nanoparticles: synthesis, functionalization, and applications in bioimaging and magnetic energy storage. Chemical Society Reviews 2009, 38 (9), 2532-2542.

12. Kim, C. H.; Myung, Y.; Cho, Y. J.; Kim, H. S.; Park, S.-H.; Park, J.; Kim, J.-Y.; Kim, B. Electronic Structure of Vertically Aligned Mn-Doped $\mathrm{CoFe}_{2} \mathrm{O}_{4}$ Nanowires and Their Application as Humidity Sensors and Photodetectors. The Journal of Physical Chemistry C 2009, 113 (17), 7085-7090.

13. Yoon, T.-J.; Lee, W.; Oh, Y.-S.; Lee, J.-K. Magnetic nanoparticles as a catalyst vehicle for simple and easy recycling. New Journal of Chemistry 2003, 27 (2), 227-229.

14. Phan, N. T. S.; Jones, C. W. Highly accessible catalytic sites on recyclable organosilane-functionalized magnetic nanoparticles: An alternative to functionalized porous silica catalysts. Journal of Molecular Catalysis A: Chemical 2006, 253 (1-2), 123-131. 15. Baruwati, B.; Guin, D.; Manorama, S. V. Pd on Surface-Modified $\mathrm{NiFe}_{2} \mathrm{O}_{4}$ Nanoparticles: A Magnetically Recoverable Catalyst for Suzuki and Heck Reactions. Organic Letters 2007, 9 (26), 5377-5380.

16. Baruwati, B.; Polshettiwar, V.; Varma, R. S. Magnetically recoverable supported ruthenium catalyst for hydrogenation of alkynes and transfer hydrogenation of carbonyl compounds. Tetrahedron Letters 2009, 50 (11), 1215-1218. 
17. Xu, S.; Feng, D.; Shangguan, W. Preparations and Photocatalytic Properties of Visible-Light-Active Zinc Ferrite-Doped $\mathrm{TiO}_{2}$ Photocatalyst. The Journal of Physical Chemistry C 2009, 113 (6), 2463-2467.

18. Fu, Y.; Chen, H.; Sun, X.; Wang, X. Combination of cobalt ferrite and graphene: High-performance and recyclable visible-light photocatalysis. Applied Catalysis B: Environmental 2012, 111-112 (0), 280-287.

19. Fu, Y.; Wang, X. Magnetically Separable $\mathrm{ZnFe}_{2} \mathrm{O}_{4}-$ Graphene Catalyst and its High Photocatalytic Performance under Visible Light Irradiation. Industrial \& Engineering Chemistry Research 2011, 50 (12), 7210-7218.

20. Lee, J.-H.; Huh, Y.-M.; Jun, Y.-w.; Seo, J.-w.; Jang, J.-t.; Song, H.-T.; Kim, S.; Cho, E.-J.; Yoon, H.-G.; Suh, J.-S.; Cheon, J. Artificially engineered magnetic nanoparticles for ultra-sensitive molecular imaging. Nat Med 2007, 13 (1), 95-99.

21. Wang, J.; Chen, Q.; Hou, B.; Peng, Z. Synthesis and Magnetic Properties of SingleCrystals of $\mathrm{MnFe}_{2} \mathrm{O}_{4}$ Nanorods. European Journal of Inorganic Chemistry 2004, 2004 (6), $1165-1168$.

22. Deng, H.; Chen, H.; Li, H. Synthesis of crystal $\mathrm{MFe}_{2} \mathrm{O}_{4}(\mathrm{M}=\mathrm{Mg}, \mathrm{Cu}, \mathrm{Ni})$ microspheres. Materials Chemistry and Physics 2007, 101 (2-3), 509-513.

23. Deng, H.; Li, X.; Peng, Q.; Wang, X.; Chen, J.; Li, Y. Monodisperse Magnetic SingleCrystal Ferrite Microspheres. Angewandte Chemie International Edition 2005, 44 (18), 27822785.

24. Yáñez-Vilar, S.; Sánchez-Andújar, M.; Gómez-Aguirre, C.; Mira, J.; SeñarísRodríguez, M. A.; Castro-García, S. A simple solvothermal synthesis of $\mathrm{MFe}_{2} \mathrm{O}_{4}(\mathrm{M}=\mathrm{Mn}, \mathrm{Co}$ and Ni) nanoparticles. Journal of Solid State Chemistry 2009, 182 (10), 2685-2690.

25. Hyeon, T.; Chung, Y.; Park, J.; Lee, S. S.; Kim, Y.-W.; Park, B. H. Synthesis of Highly Crystalline and Monodisperse Cobalt Ferrite Nanocrystals. The Journal of Physical Chemistry B 2002, 106 (27), 6831-6833.

26. Joshi, H. M.; Lin, Y. P.; Aslam, M.; Prasad, P. V.; Schultz-Sikma, E. A.; Edelman, R.; Meade, T.; Dravid, V. P. Effects of Shape and Size of Cobalt Ferrite Nanostructures on Their MRI Contrast and Thermal Activation. The Journal of Physical Chemistry C 2009, 113 (41), 17761-17767.

27. Yao, C.; Zeng, Q.; Goya, G. F.; Torres, T.; Liu, J.; Wu, H.; Ge, M.; Zeng, Y.; Wang, Y.; Jiang, J. Z. ZnFe ${ }_{2} \mathrm{O}_{4}$ Nanocrystals: Synthesis and Magnetic Properties. The Journal of Physical Chemistry C 2007, 111 (33), 12274-12278.

28. Cabrera, L.; Somoza, Á.; Marco, J.; Serna, C.; Puerto Morales, M. Synthesis and surface modification of uniform $\mathrm{MFe}_{2} \mathrm{O}_{4}(\mathrm{M}=\mathrm{Fe}, \mathrm{Mn}$, and $\mathrm{Co})$ nanoparticles with tunable sizes and functionalities. Journal of Nanoparticle Research 2012, 14 (6), 1-14.

29. Pradhan, P.; Giri, J.; Samanta, G.; Sarma, H. D.; Mishra, K. P.; Bellare, J.; Banerjee, R.; Bahadur, D. Comparative evaluation of heating ability and biocompatibility of different ferrite-based magnetic fluids for hyperthermia application. Journal of Biomedical Materials Research Part B: Applied Biomaterials 2007, 81B (1), 12-22.

30. Zhang, K.; Holloway, T.; Pradhan, A. K. Magnetic behavior of nanocrystalline $\mathrm{CoFe}_{2} \mathrm{O}_{4}$. Journal of Magnetism and Magnetic Materials 2011, 323 (12), 1616-1622.

31. Cao, X.; Gu, L. Spindly cobalt ferrite nanocrystals: preparation, characterization and magnetic properties. Nanotechnology 2005, 16 (2), 180.

32. Mazarío, E.; Herrasti, P.; Morales, M. P.; Menéndez, N. Synthesis and characterization of $\mathrm{CoFe}_{2} \mathrm{O}_{4}$ ferrite nanoparticles obtained by an electrochemical method. Nanotechnology 2012, 23 (35), 355708.

33. Liu, C.; Rondinone, A. J.; Zhang, Z. J. Synthesis of magnetic spinel ferrite $\mathrm{CoFe}_{2} \mathrm{O}_{4}$ nanoparticles from ferric salt and characterization of the size-dependent superparamagnetic properties. Pure and Applied Chemistry 2000, 72 (1-2), 37-45. 
34. Hochepied, J. F.; Bonville, P.; Pileni, M. P. Nonstoichiometric Zinc Ferrite Nanocrystals: Syntheses and Unusual Magnetic Properties. The Journal of Physical Chemistry B 2000, 104 (5), 905-912.

35. Ammar, S.; Helfen, A.; Jouini, N.; Fievet, F.; Rosenman, I.; Villain, F.; Molinie, P.;

Danot, M. Magnetic properties of ultrafine cobalt ferrite particles synthesized by hydrolysis in a polyol medium. Journal of Materials Chemistry 2001, 11 (1), 186-192.

36. Moriya, M.; Ito, M.; Sakamoto, W.; Yogo, T. One-Pot Synthesis and Morphology Control of Spinel Ferrite $\left(\mathrm{MFe}_{2} \mathrm{O}_{4}, \mathrm{M}=\mathrm{Mn}, \mathrm{Fe}\right.$, and $\left.\mathrm{Co}\right)$ Nanocrystals from Homo- and Heterotrimetallic Clusters. Crystal Growth \& Design 2009, 9 (4), 1889-1893.

37. Olsson, R. T.; Salazar-Alvarez, G.; Hedenqvist, M. S.; Gedde, U. W.; Lindberg, F.; Savage, S. J. Controlled Synthesis of Near-Stoichiometric Cobalt Ferrite Nanoparticles. Chemistry of Materials 2005, 17 (20), 5109-5118.

38. Sivakumar, P.; Ramesh, R.; Ramanand, A.; Ponnusamy, S.; Muthamizhchelvan, C. Preparation and properties of nickel ferrite $\left(\mathrm{NiFe}_{2} \mathrm{O}_{4}\right)$ nanoparticles via sol-gel autocombustion method. Materials Research Bulletin 2011, 46 (12), 2204-2207.

39. Mohapatra, S.; Rout, S. R.; Panda, A. B. One-pot synthesis of uniform and spherically assembled functionalized $\mathrm{MFe}_{2} \mathrm{O}_{4}(\mathrm{M}=\mathrm{Co}, \mathrm{Mn}, \mathrm{Ni})$ nanoparticles. Colloids and Surfaces $A$ : Physicochemical and Engineering Aspects 2011, 384 (1-3), 453-460.

40. Wang, N.; Cai, Y.; Zhang, R. Q. Growth of nanowires. Materials Science \& Engineering R-Reports 2008, 60 (1-6), 1-51.

41. Wang, Z.; Liu, X.; Lv, M.; Chai, P.; Liu, Y.; Zhou, X.; Meng, J. Preparation of OneDimensional $\mathrm{CoFe}_{2} \mathrm{O}_{4}$ Nanostructures and Their Magnetic Properties. The Journal of Physical Chemistry C 2008, 112 (39), 15171-15175.

42. Abeykoon, A. M. M.; Malliakas, C. D.; Juhás, P.; Bozin, E. S.; Kanatzidis, M. G.; Billinge, S. J. L. Quantitative nanostructure characterization using atomic pair distribution functions obtained from laboratory electron microscopes. Zeitschrift für Kristallographie Crystalline Materials 2012, 227 (5), 248-256.

43. Chupas, P. J.; Qiu, X.; Hanson, J. C.; Lee, P. L.; Grey, C. P.; Billinge, S. J. L. Rapid Acquisition Pair Distribution Function Analysis. Journal of Applied Crystallography 2003, $36,1342-1347$.

44. Hammersley, A. P.; Svenson, S. O.; Han, M.; Hauserman, D. Two-dimensional detector software: From real detector to idealised image or two-theta scan. High Pressure Research 1996, 14, 235-248.

45. Juhas, P.; Davis, T.; Farrow, C. L.; Billinge, S. J. L. PDFgetX3: A rapid and highly automatable program for processing powder diffraction data into total scattering pair distribution functions. Journal of Applied Crystallography 2013, 46, 560-566.

46. Egami, T.; Billinge, S. J. L. Underneath the Bragg peaks: structural analysis of complex materials. 2nd. ed.; Elsevier: Amsterdam, 2012.

47. Proffen, T.; Billinge, S. J. L. PDFFIT a program for full profile structural refinement of the atomic pair distribution function. Journal of Applied Crystallography 1999, 32, 572575 .

48. Farrow, C. L.; Juhas, P.; Liu, J.; Bryndin, D.; Bozin, E. S.; Bloch, J.; Pro, T.; Billinge, S. J. L. PDFfit2 and PDFgui: computer programs for studying nanostructure in crystals. Journal of Physics: Condensed Matter 2007, 19 (33), 335219.

49. Abeykoon, A. M. M.; Hu, H.; Wu, L.; Zhu, Y.; Billinge, S. J. L. Calibration and data collection protocols for reliable lattice parameter values in electron pair distribution function studies. Journal of Applied Crystallography 2015, 48, 244-251.

50. Farrow, C. L.; Juhas, P.; Billinge, S. J. L. SrFit. 2010, unpublished.

51. Farrow, C. L.; Billinge, S. J. L. Relationship between the atomic pair distribution function and small-angle scattering: implications for modeling of nanoparticles. Acta Crystallographica 2009, 65, 232. 
52. Shi, C.; Redmond, E. L.; Mazaheripour, A.; Juhas, P.; Fuller, T. F.; Billinge, S. J. L. Anomalous bond softening and disorder below $2 \mathrm{~nm}$ diameter in carbon supported platinum nanoparticles from the temperature dependent peak width of the atomic pair distribution function. Journal of Physical Chemistry C 2013, 117, 7226-7230.

53. Masadeh, A. S.; Bozin, E. S.; Farrow, C. L.; Paglia, G.; Juhas, P.; Karkamkar, A.; Kanatzidis, M. G.; Billinge, S. J. L. Quantitative size-dependent structure and strain determination of $\mathrm{CdSe}$ nanoparticles using atomic pair distribution function analysis. Physical Review B 2007, 76 (11), 115413/1 - 115413/11.

54. Gyergyek, S.; Drofenika, M.; Makovec, D. Oleic-acid-coated $\mathrm{CoFe}_{2} \mathrm{O}_{4}$ nanoparticles synthesized by co-precipitation and hydrothermal synthesis. Materials Chemistry and Physics 2012, 133 (1), 515-522.

55. Papaefthymiou, G. C.; Devlin, E.; Simopoulos, A.; Yi, D. K.; Riduan, S. N.; Lee, S. S.; Ying, J. Y. Interparticle interactions in magnetic core/shell nanoarchitectures. Physical Review B 2009, 80 (2), 024406.

56. Mørup, S.; Oxborrow, C. A.; Hendriksen, P. V.; Pedersen, M. S.; Hanson, M.; Johansson, C. Magnetic and mechanical coupling between ultrafine maghemite particles. Journal of Magnetism and Magnetic Materials 1995, 140-144, Part 1 (0), 409-410. 57. Mørup, S. Superparamagnetism and Spin Glass Ordering in Magnetic Nanocomposites. EPL (Europhysics Letters) 1994, 28 (9), 671.

58. Luo, W.; Nagel, S. R.; Rosenbaum, T. F.; Rosensweig, R. E. Dipole interactions with random anisotropy in a frozen ferrofluid. Physical Review Letters 1991, 67 (19), 2721-2724.

59. Papaefthymiou, G. C. Nanoparticle magnetism. Nano Today 2009, 4 (5), 438-447.

60. Roca, A. G.; Carmona, D.; Miguel-Sancho, N.; Bomatí-Miguel, O.; Balas, F.; Piquer, C.; Santamaría, J. Surface functionalization for tailoring the aggregation and magnetic behaviour of silica-coated iron oxide nanostructures. Nanotechnology 2012, 23 (15), 155603/1-10.

61. Bala, T.; Sankar, C. R.; Baidakova, M.; Osipov, V.; Enoki, T.; Joy, P. A.; Prasad, B. L. V.; Sastry, M. Cobalt and Magnesium Ferrite Nanoparticles: Preparation Using Liquid Foams as Templates and Their Magnetic Characteristics. Langmuir 2005, 21 (23), 1063810643.

62. Wan, J.; Jiang, X.; Li, H.; Chen, K. Facile synthesis of zinc ferrite nanoparticles as non-lanthanide $\mathrm{T}_{1}$ MRI contrast agents. Journal of Materials Chemistry 2012, 22 (27), 1350013505.

63. Zhang, H.-g.; Zhang, Y.-J.; Wang, W.-H.; Wu, G.-H. Origin of the constricted hysteresis loop in cobalt ferrites revisited. Journal of Magnetism and Magnetic Materials 2011, 323 (15), 1980-1984.

64. Papaefthymiou, G. C.; Ahmed, S. R.; Kofinas, P. Magnetic and Structural Characterization of $\mathrm{CoFe}_{2} \mathrm{O}_{4}$ nanoparticles encapsulated within block copolymer films. Reviews on Advanced Materials Science 2005, 10 (4), 306-313.

65. Sawatzky, G. A.; Van Der Woude, F.; Morrish, A. H. Cation Distributions in Octahedral and Tetrahedral Sites of the Ferrimagnetic Spinel $\mathrm{CoFe}_{2} \mathrm{O}_{4}$. Journal of Applied Physics 1968, 39 (2), 1204-1205.

66. Papaefthymiou, G. C. Nanostructural Studies by Mössbauer Spectroscopy. MRS Online Proceedings Library 1994, 332.

67. Néel, L. Theorie du trainage magnetique des ferromagnetiques en grains fins avec applications aux terres cuites. Annals of Geophysics 1949, 5, 99-136.

68. Brown, W. F., Jr. Thermal Fluctuations of a Single-Domain Particle. Physical Review 1963, 130 (5), 1677-1686.

69. Brown, J. W. F. The Fundamental Theorem of Fine-Ferromagnetic-Particle Theory. Journal of Applied Physics 1968, 39 (2), 993-994. 
70. Aharoni, A. Relaxation Processes in Small Particles. In Magnetic Properties of Fine Particles, Dormann, J. L.; Fiorani, D., Eds. Elsevier Science: North-Holland, 1991; pp 3-12. 71. Cullity, B. D.; Graham, C. D. Introduction to Magnetic Materials. 2nd ed.; John Wiley \& Sons, Inc.: Hoboken, 2009.

72. Aharoni, A. Introduction to the Theory of Ferromagnetism. 2nd ed.; Oxford University Press: Oxford, 2007.

73. Monson, T. C.; Venturini, E. L.; Petkov, V.; Ren, Y.; Lavin, J. M.; Huber, D. L. Large enhancements of magnetic anisotropy in oxide-free iron nanoparticles. Journal of Magnetism and Magnetic Materials 2013, 331 (0), 156-161.

74. Bødker, F.; Mørup, S.; Linderoth, S. Surface effects in metallic iron nanoparticles. Physical Review Letters 1994, 72 (2), 282-285.

75. Dormann, J. L.; Fiorani, D., Eds. Magnetic Properties of Fine Particles. Elsevier Science: North-Holland, 1991.

76. Coey, J. M. D. Magnetism and Magnetic Materials. Cambridge University Press: Cambridge, UK, 2012.

77. Rondinone, A. J.; Samia, A. C. S.; Zhang, Z. J. Characterizing the magnetic anisotropy constant of spinel cobalt ferrite nanoparticles. Applied Physics Letters 2000, 76 (24), 36243626 .

78. Kim, D.; Lee, N.; Park, M.; Kim, B. H.; An, K.; Hyeon, T. Synthesis of Uniform Ferrimagnetic Magnetite Nanocubes. Journal of the American Chemical Society 2008, 131 (2), 454-455.

79. Pradeep, A.; Priyadharsini, P.; Chandrasekaran, G. Sol-gel route of synthesis of nanoparticles of $\mathrm{MgFe}_{2} \mathrm{O}_{4}$ and XRD, FTIR and VSM study. Journal of Magnetism and Magnetic Materials 2008, 320 (21), 2774-2779.

80. $\quad$ Šepelák, V.; Bergmann, I.; Menzel, D.; Feldhoff, A.; Heitjans, P.; Litterst, F. J.; Becker, K. D. Magnetization enhancement in nanosized $\mathrm{MgFe}_{2} \mathrm{O}_{4}$ prepared by mechanosynthesis. Journal of Magnetism and Magnetic Materials 2007, 316 (2), e764-e767. 81. Upadhyay, C.; Verma, H. C.; Sathe, V.; Pimpale, A. V. Effect of size and synthesis route on the magnetic properties of chemically prepared nanosize $\mathrm{ZnFe}_{2} \mathrm{O}_{4}$. Journal of Magnetism and Magnetic Materials 2007, 312 (2), 271-279.

82. Schiessl, W.; Potzel, W.; Karzel, H.; Steiner, M.; Kalvius, G. M.; Martin, A.; Krause, M. K.; Halevy, I.; Gal, J.; Schäfer, W.; Will, G.; Hillberg, M.; Wäppling, R. Magnetic properties of the $\mathrm{ZnFe}_{2} \mathrm{O}_{4}$ spinel. Physical Review B 1996, 53 (14), 9143-9152.

83. Jiang, J. Z.; Goya, G. F.; Rechenberg, H. R. Magnetic properties of nanostructured $\mathrm{CuFe}_{2} \mathrm{O}_{4}$. Journal of Physics: Condensed Matter 1999, 11 (20), 4063.

84. Nakhjavan, B.; Tahir, M. N.; Panthofer, M.; Gao, H.; Schladt, T. D.; Gasi, T.; Ksenofontov, V.; Branscheid, R.; Weber, S.; Kolb, U.; Schreiber, L. M.; Tremel, W. Synthesis, characterization and functionalization of nearly mono-disperse copper ferrite $\mathrm{Cu}_{\mathrm{x}} \mathrm{Fe}_{3-\mathrm{x}} \mathrm{O}_{4}$ nanoparticles. Journal of Materials Chemistry 2011, 21 (19), 6909-6915. 85. Laokul, P.; Amornkitbamrung, V.; Seraphin, S.; Maensiri, S. Characterization and magnetic properties of nanocrystalline $\mathrm{CuFe}_{2} \mathrm{O}_{4}, \mathrm{NiFe}_{2} \mathrm{O}_{4}, \mathrm{ZnFe}_{2} \mathrm{O}_{4}$ powders prepared by the Aloe vera extract solution. Current Applied Physics 2011, 11 (1), 101-108.

86. Bao, N.; Shen, L.; Wang, Y.; Padhan, P.; Gupta, A. A Facile Thermolysis Route to Monodisperse Ferrite Nanocrystals. Journal of the American Chemical Society 2007, 129 (41), 12374-12375.

87. Issa, B.; Obaidat, I. M.; Albiss, B. A.; Haik, Y. Magnetic nanoparticles: surface effects and properties related to biomedicine applications. International Journal of Molecular Sciences 2013, 14 (11), 21266-21305.

88. Kale, A.; Gubbala, S.; Misra, R. D. K. Magnetic behavior of nanocrystalline nickel ferrite synthesized by the reverse micelle technique. Journal of Magnetism and Magnetic Materials 2004, 277 (3), 350-358. 
89. Chinnasamy, C. N.; Narayanasamy, A.; Ponpandian, N.; Chattopadhyay, K.; Shinoda, K.; Jeyadevan, B.; Tohji, K.; Nakatsuka, K.; Furubayashi, T.; Nakatani, I. Mixed spinel structure in nanocrystalline $\mathrm{NiFe}_{2} \mathrm{O}_{4}$. Physical Review B 2001, 63 (18), 184108/1-184108/6. 90. Billinge, S. J. L.; Levin, I. The Problem with Determining Atomic Structure at the Nanoscale. Science 2007, 316 (5824), 561-565.

91. Dinnebier, R. E.; Billinge, S. J. L. Powder Diffraction: Theory and Practice. 1 ed.; Royal Society of Chemistry Cambridge, UK, 2008.

92. Tripathi, S.; Petkov, V.; Selbach, S. M.; Bergum, K.; Einarsrud, M.-A.; Grande, T.; Ren, Y. Structural coherence and ferroelectric order in nanosized multiferroic YMnO3. Physical Review B 2012, 86 (9), 094101/1-094101/8.

93. Jovic, N. G.; Masadeh, A. S.; Kremenovic, A. S.; Antic, B. V.; Blanusa, J. L.; Cvjeticanin, N. D.; Goya, G. F.; Antisari, M. V.; Bozin, E. S. Effects of Thermal Annealing on Structural and Magnetic Properties of Lithium Ferrite Nanoparticles. Journal of Physical Chemistry C 2009, 113 (38), 20559-20567.

94. Kumar, L.; Kumar, P.; Narayan, A.; Kar, M. Rietveld analysis of XRD patterns of different sizes of nanocrystalline cobalt ferrite. International Nano Letters 2013, 3 (1), 8(1)(8)12.

95. Gateshki, M.; Petkov, V.; Pradhan, S. K.; Vogt, T. Structure of nanocrystalline $\mathrm{MgFe}_{2} \mathrm{O}_{4}$ from X-ray diffraction, Rietveld and atomic pair distribution function analysis Journal of Applied Crystallography 2005, 38, 772-779.

96. Li, J.; Liu, Z.; Zhu, Z. Magnetically separable $\mathrm{ZnFe}_{2} \mathrm{O}_{4}, \mathrm{Fe}_{2} \mathrm{O}_{3} / \mathrm{ZnFe}_{2} \mathrm{O}_{4}$ and $\mathrm{ZnO} / \mathrm{ZnFe}_{2} \mathrm{O}_{4}$ hollow nanospheres with enhanced visible photocatalytic properties. $R S C$ Advances 2014, 4 (93), 51302-51308.

97. Mishra, D.; Senapati, K. K.; Borgohain, C.; Perumal, A. Magnetic Nanocomposites as Photocatalyst for the Degradation of Methyl Orange Dye. Journal of Nanotechnology 2012, 323145/1-323145/6.

98. Gao, L.; Zhuang, J.; Nie, L.; Zhang, J.; Zhang, Y.; Gu, N.; Wang, T.; Feng, J.; Yang, D.; Perrett, S.; Yan, X. Intrinsic peroxidase-like activity of ferromagnetic nanoparticles. Nature Nanotechnology 2007, 2, 577-583.

99. Shi, W.; Zhang, X.; He, S.; Huang, Y. $\mathrm{CoFe}_{2} \mathrm{O}_{4}$ magnetic nanoparticles as a peroxidase mimic mediated chemiluminescence for hydrogen peroxide and glucose. Chemical Communications 2011, 47 (38), 10785-10787.

100. Su, L.; Feng, J.; Zhou, X.; Ren, C.; Li, H.; Chen, X. Colorimetric Detection of Urine Glucose Based $\mathrm{ZnFe}_{2} \mathrm{O}_{4}$ Magnetic Nanoparticles. Analytical Chemistry 2012, 84 (13), 5753 5758.

101. Liu, C.; Zhang, Z. J. Size-dependent superparamagnetic properties of Mn spinel ferrite nanoparticles synthesized from reverse micelles. Chemistry of Materials 2001, 13, 2092-2096. 102. Moumen, N.; Pileni, M. P. New Syntheses of Cobalt Ferrite Particles in the Range of 2-5 nm: Comparison of the Magnetic Properties of the Nanosized Paticles in Dispersed Fluid or in Powder Form. Chemistry of Materials 1996, 8, 1128-1134. 